\title{
Physiologic constraints of using exosomes in vivo as systemic delivery vehicles
}

\author{
Dong Soo Lee ${ }^{1,2^{*}}$, Minseok Suh $^{2}$, Seo Young Kang ${ }^{2,3}$, Do Won Hwang ${ }^{1}$ \\ ${ }^{1}$ Department of Nuclear Medicine, Seoul National University Hospital, Seoul, Republic of Korea \\ ${ }^{2}$ Department of Molecular Medicine and Biopharmaceutical Sciences, Graduate School of Convergence \\ Science and Technology, Seoul National University, Seoul, Republic of Korea \\ ${ }^{3}$ Department of Nuclear Medicine, Ewha Womans University Medical Center, Seoul, Republic of Korea
}

Submitted: July 8, 2019

Accepted: July 29, 2019

Published: July 30, 2019

\section{Abstract}

Systemic delivery of exosomes meets hurdles which had not been elucidated using live molecular imaging for their biodistribution. Production and uptake of endogenous exosomes are expected to be nonspecific and specific, respectively, where external stimuli of production of exosomes and their quantitative degree of productions are not understood. Despite this lack of understanding of basic physiology of in vivo behavior of exosomes including their possible paracrine or endocrine actions, many engineering efforts are taken to develop therapeutic vehicles. Especially, the fraction of exosomes' taking the routes of waste disposal and exerting target actions are not characterized after systemic administration. Here, we reviewed the literature about in vivo distribution and disposal/excretion of exogenous or endogenous exosomes and, from these limited resources of knowledge currently available, summarized the knowledge and the uncertainties of exosomes on physiologic standpoints. An eloquent example of the investigations to understand the roles and confounders of exosomes' action in the brain was highlighted with emphasis on the recent discovery of brain lymphatics and hypothesis of glymphatic/lymphatic clearance pathways in diseases as well as in physiologic processes. The possibility of delivering therapeutic exosomes through the systemic circulation, across blood-brain barriers and finally to target cells such as microglia, astrocytes and/or neurons is a good testbed in which the investigators can formulate problems to solve for both understanding (science) and application (engineering).

\section{Keywords}

Extracellular vesicles, Exosomes, Biodistribution, Waste disposal, Live imaging, Brain exosomes

\section{Purpose and rationale}

In this review, we emphasize the pharmacological and/or physiologic aspects of systemically delivered exogenous exosomes in vivo.(1-3) We review the core ideas incipient in the scientific community, the hyped suggestions and implications of investigators in materials sciences and biopharmaceutical sciences.(4-16) People tend to think, that if novel bio/nanomaterials work in vitro, they are sure will be working similarly in vivo. However, once injected in the body systemically through intravenous or intraperitoneal routes, the materials meet serum proteins ("corona"), immune cells (both innate and adaptive), narrow capillaries and their endothelial cells, those one of the nonprofessional phagocytic cells. These obstacles should be considered, and bio/nanomaterials must be modified accordingly.

\section{Summary of Relevant Literature and Discussion}

Extracellular vesicles include exosomes, ectosomes, microvesicles, and apoptotic bodies. Exosomes are sometimes used as an

* Corresponding author: Prof. Dong-Soo Lee, MD, PhD., Dept of Nuclear Medicine, Seoul National University Hospital, 101 Daehak-ro Jongnogu Seoul, 110-744 Korea Tel: 82-2-2072-2501 Fax; 82-2745-7690 Email: ds1@plaza.snu.ac.kr 
alias name for extracellular vesicles.(17-19) There were hopes and sincere trials to use exosomes as therapeutic vehicles, though investigators did not understand the specificity of production (and release) and the specificity of uptake (and the action on the recipient cells) very well.(19-22) Instead, there were speculations that the release was not supposed to be specific(17) though the fates of the released exosomes were not well traced locally in the microenvironment or remotely via circulations.(18) Paracrine and endocrine mechanism of actions can be an analogy for these intercellular communications via exosomes. Therapeutic efficacy of exogenous exosomes had been presumed without any evidence not to differ between paracrine and endocrine actions of released (and taken up) exosomes.

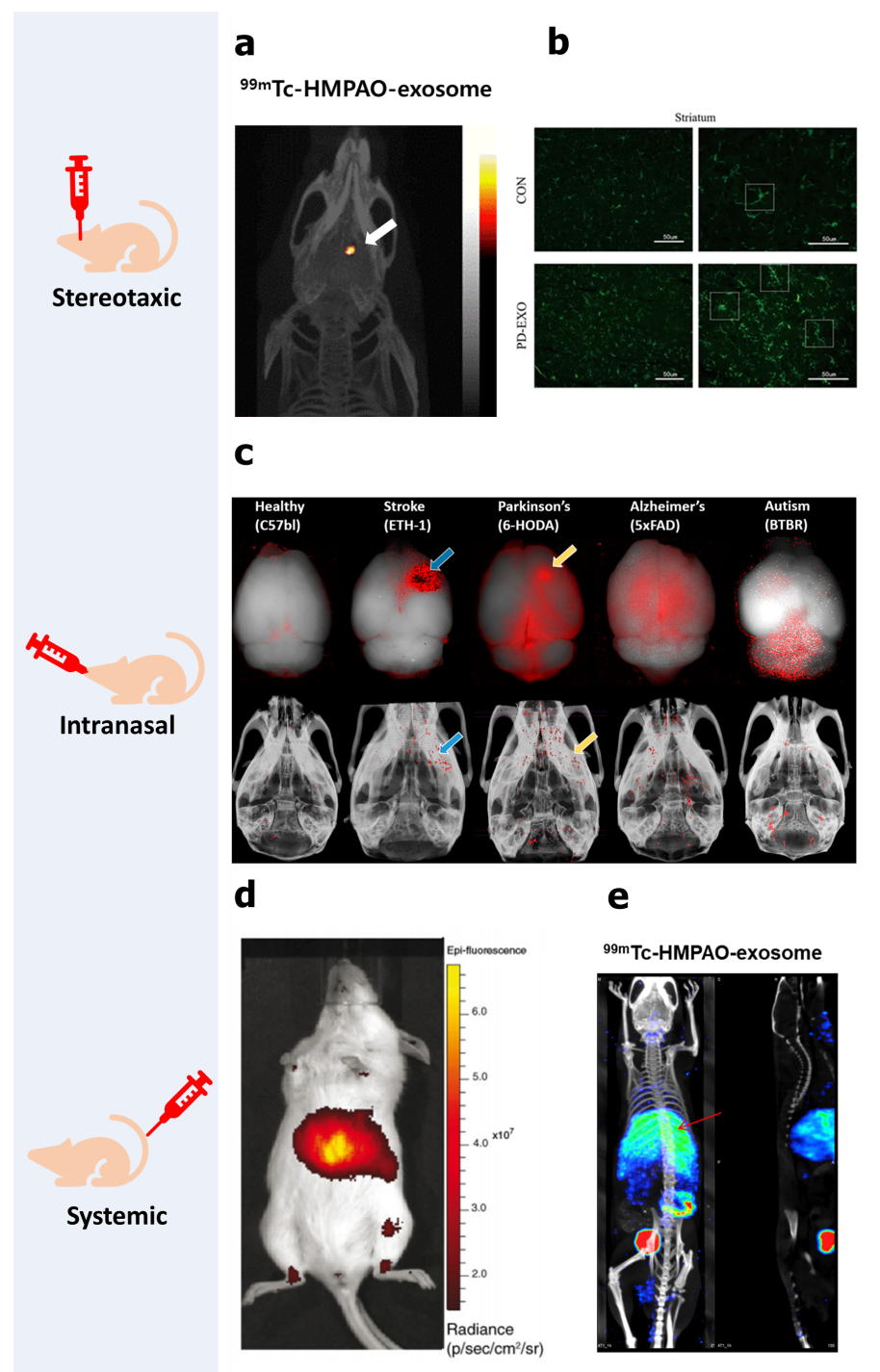

Figure 1. Different routes for administration of exogenous exosomes. (a) Exosomes isolated from mouse plasma was labeled with $99 \mathrm{mTc}$-HMPAO. The intracranial injection of $99 \mathrm{mTc}$-HMPAO-labeled exosomes clearly showed the exosome distribution (white arrow) in the injected site of the brain at 2 hours post-injection measured by SPECT/CT (preliminary result from our group). (b) Stereotaxic administration of exosomes modulated alpha-synuclein transmission by dysregulating microglia (reproduced with permission from reference 4). (c) Different distribution patterns of exosomes were identified in various brain pathologies after intranasal administration. Blue and the yellow arrow indicates the injection site of synthetic compounds for the model generation (reproduced with permission from reference 25). (d) Representative IVIS image showing 24-hour biodistribution of exogenous exosomes after systemic administration (reproduced with permission from reference 3). (e) Exosomes released from human breast cancer cell line was isolated using ultracentrifugation. 99mTc-HMPAO-exosomes were prepared after purification using the spin column to remove non-labeled free 99mTc-HMPAO. 99mTc-HMPAO-exosomes were intravenously administered to the mouse. SPECT/CT image was obtained at 1 hour after injection. Red arrow indicates the liver tissue of mouse injected with 99mTc-HMPAO-exosomes (preliminary result from our group). 
Successful in vitro experiments have been hastily extrapolated to the expectation that the therapeutic effect of exogenous exosomes would be exerted to the recipient cells.(3-16) However, it might not be the case with exosomes as the success of the application of biopharmaceuticals to in vivo and human use was slow though biopharmaceutical sciences had been so successful during the last several decades, and investigators should have overcome many hurdles and barriers. Stereotaxic $(23,24)$ or intratumoral injection of exosomes or their delivery via the intranasal route(25) are practically not the final solutions but the first step of a long journey of producing clinically usable forms of exosomes which would be delivered systemically (Figure 1).

Investigation of the plausibility of using exogenous exosomes by systemic administration should be accompanied by an investigation of in vivo whereabouts using trustworthy tracing methods. $(26,27)$ In vivo molecular imaging is the best and radionuclide labeling of exosomes or fluorescent dye or reporter proteins labeling of exosomes were proposed to meet these needs. $(1,26-27)$ In any of these cases, securing the stability of labels was the most important here in tracing the pharmacokinetics of systemically delivered exogenous exosomes.(28) Pharmacokinetics and pharmacodynamics studies using molecular imaging are also of the utmost importance in the investigation of assessing the therapeutic usefulness of exogenous exosomes.

For biodistribution/excretion and molecular imaging studies, we need a priori to know the details of the biology of exosomes production and release, exosomes physiology and pharmacology and biology of uptakes and effects upon target (or recipient) cells.(17-22) These fields of research had advanced to reveal many details recently enabling us to understand the possible mechanism which we might hitchhike the pathways of uptake and the consequent action on the target cells after successful targeted delivery through systemic administration. There are several excellent reports which explained the helpful details of the interaction of exosomes with the target cells and indicative perspective reports to enlighten the importance of the intermediaries delivering exosomes via circulation.(17-22) For this review, we collected and edited the knowledge available in this field to let the readers see easily the importance of finding the whereabouts of exogenous exosomes and based on this, improving the probability of delivering the exosomes to the target organs and cells. Uptake and plausible therapeutic action of exosomes would lead us to predict the expected efficacy of exosomes upon the target cells.

When novel bio/nanomaterials are injected in the body systemically through intravenous or intraperitoneal routes, the materials meet serum proteins called as corona,(29-31) both innate and adaptive immune cells,(32) and narrow capillaries and their endothelial cells, one of the non-professional phagocytic cells. These obstacles must be considered, and the bio/nanomaterials must be modified accordingly.

In addition, there is a common misunderstanding that liver uptakes are automatically in the macrophage phagocytic system (MPS) while ignoring the possibility that they supply routes of excretion.(33) Another trap was the ignorance or misunderstanding that the excretion or the clearance of exogenous exosomes in systemic circulation was not always physiologic and thus should be or can be overcome by engineering. But, do we know the real significance of systemic circulation of exosomes and thus endocrine action of endogenous exosomes(27) or whether the excreted exosomes are born to be wasted like the endogenous exosomes or not?

The hype of wishful therapeutic effects of exosomes

Nanomedicine is the medical application of nanotechnology for various purposes. For using nanomaterials as possible therapeutics/ diagnostics in vivo, nanomedicine is using nanomedicines for therapy and imaging. Nanomedicines consists of endogenous and exogenous nanomaterials produced in vivo or ex vivo, respectively. $(33,34)$ Exosomes can be considered as endogenous nanomaterials. However, once exosomes are produced ex vivo and scaled up to reach the volume of systemic administration, they resemble the status of the monoclonal antibodies produced at industrial scale(35) in that they face the problems such as chemical engineering, mixing, separation and 
purification complying with good manufacturing practice or the differentiated retinal pigment epithelium (RPE) cells sheet made from induced pluripotent stem cell (iPSC).(36, 37) Monoclonal antibodies might provoke innate and adaptive immune responses and thus blood clearance was short. The second (or even the first) administration did not meet the purpose of maintaining sufficiently long circulation time $(38,39)$. In particular, antibody-drug conjugates have always raised concerns about labeling efficacy.(40, 41) Antimouse antibody response has been well recognized for mouse monoclonal antibodies, but anti-human antibody response may also obliterate the efficacy of humanized or human monoclonal antibody therapy.

Exosomes can experience the same fate, but few were reported(26, 27) and just the speculations prevail about the fate of systemically administered exosomes. Exosomes are known to have many membrane proteins on the surface including major histocompatibility complex (MHC) molecules. Strangely enough, allogeneic exosomes have not been considered to be immunogenic even without evidence of non-immunogenicity. Absence of evidence of immunogenicity does not mean an absence of immunogenicity. However, investigators tend not to use xenogeneic exosomes for testing the efficacy in vitro if non-xenogeneic exosomes are available, and they just don't emphasize this.

Differentiated RPE was first proposed to be used as therapy after iPSC generation from autologous fibroblast and their consequent differentiation to pigment epithelium.(36) As was expected, it caused much inconvenience to use autologous cells and took time to process the transplants. As expected, allogeneic differentiated RPE was produced using donor fibroblasts, pre-manufactured and ready for transplantation. Unfortunately, recent reports say that allogeneic differentiated RPE caused serious immune responses.(37) Likewise, as exosomes have MHC1 and MHC2 molecules, they will meet immune cells during circulation and cause immune cells-mediated clearance.

The possible immune response against the systemically administered exosomes is unpredictable. This difficulty of predicting immune responses to exogenous exosomes are derived from (a) the difficulty of characterizing the surface MHC molecules of individual exosomes due to the necessity of unrealistic mass spectrometry, which requires the millionfold higher sensitivity than the currently available ones, (b) the inability to view the interaction of the exosomes with the relevant immune cells such as monocytes, neutrophils and tissue-resident macrophages on real-time, though this has become possible in transparent zebrafish. $(26,27)$ and (c) lack of knowledge of exact uptake mechanism of exosomes to the relevant cells such as dendritic cells, monocytes, macrophages or other nonprofessional phagocytic cells. The nonprofessional phagocytic cells include endothelial cells, especially in the lung capillaries, the liver sinusoidal endothelial cells, and hepatocytes which would be important in clearing exosomes in vivo. In both capillaries and sinusoids, endothelial cells and associated tissue-resident macrophages have enough time to process exosomes.

Clearance from the blood is the single most important step of maintaining the balance between the production of exosomes by the donor cells and their uptake and degradation by the recipient cells (and action in some cases). The clearance of apoptotic bodies are well known as 200-300 billion cells (200-300 grams of cells) are recycled daily in humans(42) and microvesicles are the next ones to be cleared from the site of production or from the circulation and the recipient cells. Once in the circulation, they are prone to be taken up by circulating or resident phagocytic cells. However, the exosomes (or ectosomes) do sneak easily into the space of Disse in case of the liver through the fenestra (size of $100 \mathrm{~nm}$ ) of the liver sinusoidal endothelium.(33, 43) Hepatocytes meet the exosomes directly and thus the liver uptake should be cautiously considered as MPS uptake. Liver uptake of exosomes is more likely to be due to the hepatocytes since we can observe intestinal activity in the following images overtimes $(2,4$, 6 or 12 hours after administration).(33, 44) 


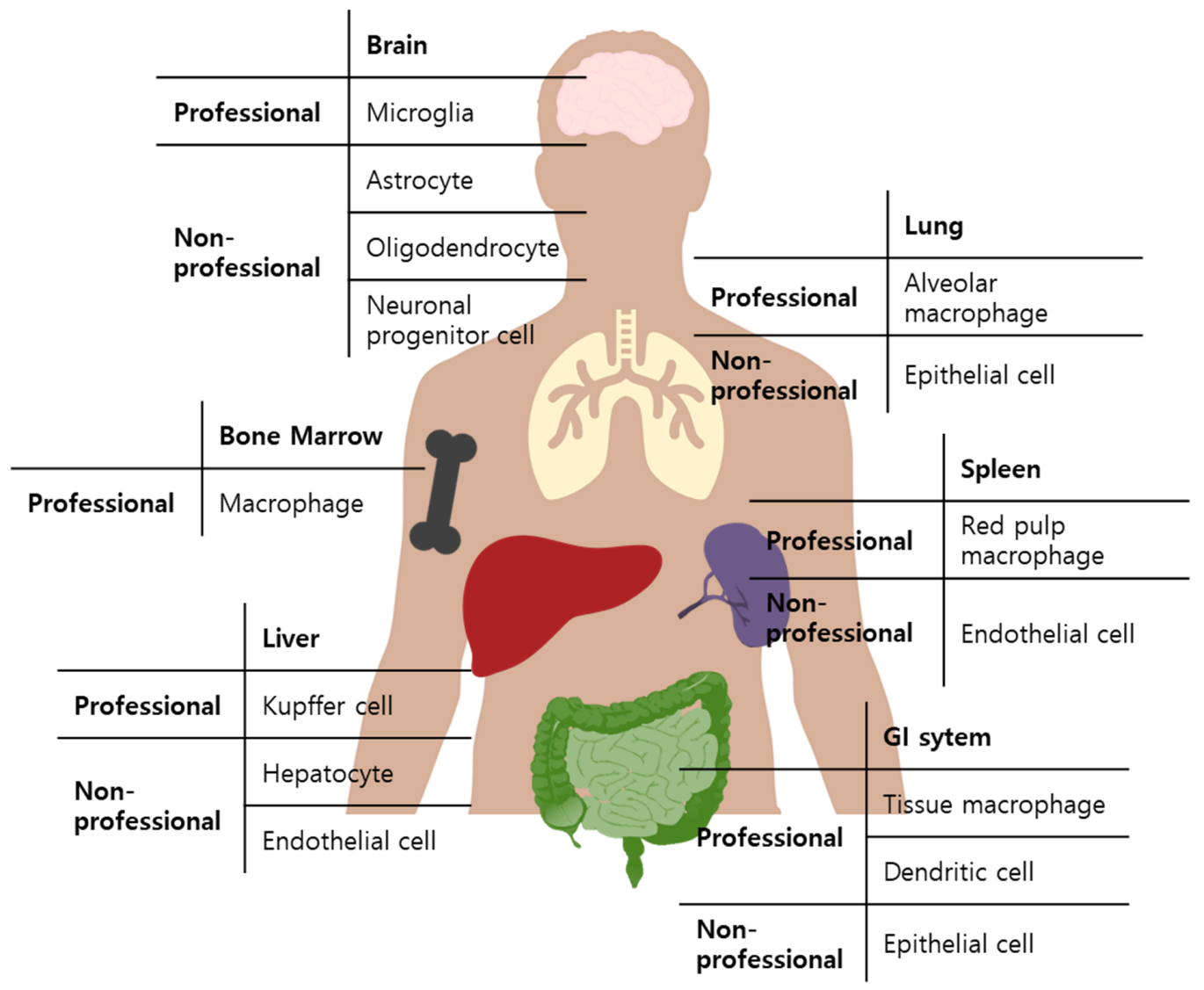

Figure 2. Summary of possible clearance site of exosomes via professional and non-professional phagocytes.

If it decays in situ in the liver, we can suspect that exosomes were cleared by Kupffer cells or non-professional phagocytic cells of the liver such as liver sinusoidal endothelial cells (Figure 2)

To guarantee the plausibility using exogenous exosomes as therapeutics for various diseases, elucidation of pharmacokinetics, biodistribution, and also the excretion must be preceded. Most important pathways through liver and kidneys are the ones of desired attention, however, the interaction of exosomes with circulating phagocytic cells, capillary endothelial cells and hepatocytes should be also paid attention to.

\section{Targeted delivery of exogenous exosomes administered systemically}

There have been several reports to insist that engineering the exosomes was successful for targeted delivery and the exosomes should have been modified and prepared as therapeutics in their proposed ways. The first report was the mimicry of intracoronary artery delivery by using isolated rabbit heart model. In this ex vivo model, Lim and her colleagues(45) could deliver the exosomes prepared ex vivo to the ligated-and-reperfused coronary arteries and found that anti-apoptotic clusters and glycolytic apparatus of peptides were delivered so that the reperfused myocardial territories were improved in their function. Scale-up of the production of therapeutic exosomes were proposed by the authors. A recent study(46) demonstrated a novel targeting method using cardiac homing peptide (Figure 3A). After systemic administration it showed successful outcomes, reducing fibrosis and scar size, and increasing cellular regeneration, in myocardial infarction model. 


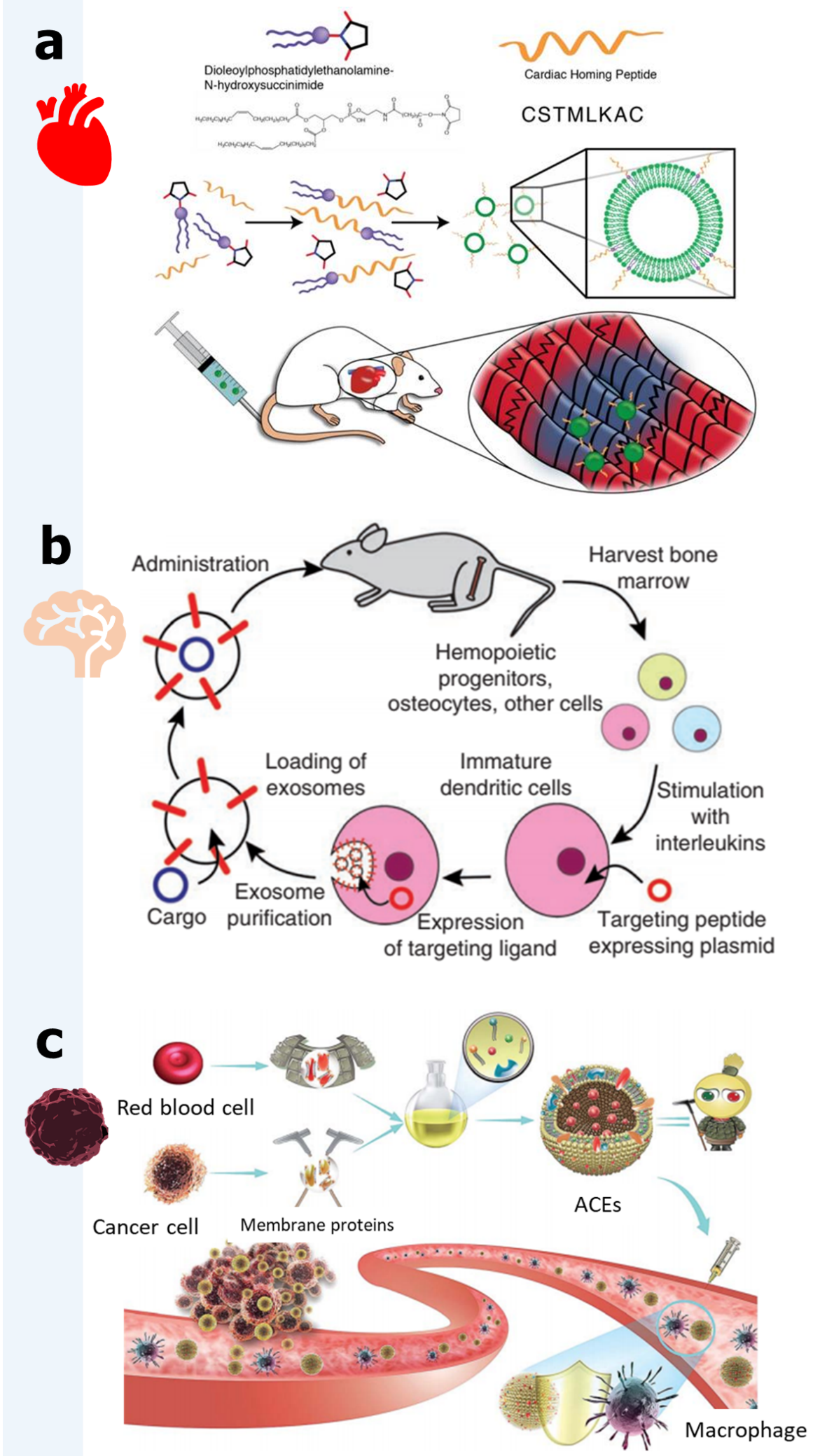

Figure 3. Studies regarding successful targeted delivery of exosomes. (a) Targeting strategy using cardiac homing peptide showed a promising outcome in the myocardial infarction model after systemic exosome administration (reproduced with permission from reference 46). (b) Schematic illustration of production, engineering, and administration of exogenous exosomes derived from rabies glycopeptide-expressed dendritic cell. After systemic administration, engineered exogenous exosomes were delivered to neurons, microglia, and oligodendrocytes in the brain (reproduced with permission from reference 47). (c) Engineering of exosomes to avoid the surveillance of phagocytic cells and to specifically target tumor showed promising results (reproduced with permission from reference 48). 
The second important one was the successful delivery of exogenous exosomes derived from rabies glycopeptide-expressed dendritic cells.(47) The exosomes were identified to have rabies glycopeptide and thus showed the targeting capability to the brain (Figure 3B). The authors administered the manufactured exosomes intravenously and insisted that the exosomes reached the brain via circulation and crossed the blood-brain barrier (BBB) and reached the target cells in the brain.

The recent more popular successes used to be reported for the investigators' success of making the exosomes hidden from the surveillance of phagocytic cells and to find the targets in the tumors (Figure 3C). Investigators made artificial chimeric exosomes, based on liposome combined with cell surface molecules, and treated them with enzymes to prove their biological effects. They even made artificial chimeric exosomes with the capability of sending SIRP-1 signals to the immune cells while preserving targeting capability.(48) The investigators didn't modify the surface, but implied the possible clinical use in humans(49, 50); however, as is well known, although modification is possible for many sites, this makes it less likely to be translatable for use in clinics.

Preparation of exosomes in vitro from the cells was the bottleneck and the progress take place in the development of the fast methods or the improvement of the yield without the deterioration of the characteristics of the exosomes. Regarding the former goal of the fast and agile method, investigators introduced exosome-mimetic nanovesicles, which were made by passing the cells serially through the microchannels of diameters of $5 \mu \mathrm{m}$ to 1 $\mu \mathrm{m}$. (51-55) Interestingly, this endeavor made exosome-mimetics have surface markers of CD63, CD81 or Alix and others. The functional verification was not comprehensive, but the proof of concept studies was only done especially with the first report by Gho and his colleagues; however, the authors could successfully deliver exosomes to tumors after systemic administration.(52)

One of the most recently reported successes was interesting as the authors used the intraperitoneal route of exosomes as a delivery method. The SIRP-1 modified exosomes(48, 56) were used for intraperitoneal injection and the appearance of exosomes' fluorescence in blood was just used as evidence of systemic delivery of exosomes using this method. In this investigation, tumor targeting was the goal of the administration of exosomes. Missing was the information regarding the pharmacokinetic/biodistribution, targeted delivery, and pharmacodynamics.

Difference between paracrine release and endocrine circulation of exosomes

The paracrine mediator acts on nearby cells via extracellular spaces while endocrine mediator, hormones, act on remote cells via the circulatory system. The reason for the existence of exosomes released from tissues should be understood at the local milieu of interstitial spaces.(25) Interstitial space is not void space but with full of local intercellular messengers including exosomes (Figure 4). Exosomes are released and can go anywhere without resistances as they can float over the fluidic milieu due to the advection, convection, and diffusion processes. Biophysical forces driving the dispersion of exosomes and other particulate materials have been reported. Unlike cells, exosomes do not have selfmobility and mimic the immotile enveloped viruses. Enveloped viruses mimic exosomes in the shape and mobility between cells. Peptide or lipid mediators are much smaller ones which mostly take diffusion processes and the uptake to the target cells via receptor-mediated touch action or endocytosis. Antibodies with the size of $12-15 \mathrm{~nm}$ are the quite familiar examples of these macromolecules as peptides, or more exactly, glycopeptides. Other cytokines such as interleukins or interferons are another example. Lipid mediators such as prostaglandins, leukotrienes, or 5-HETE/12-HETE are much smaller counterparts but function in the same way as their peptide counterparts. All these molecules are considered to act as paracrine actors. Interestingly, paracrine action of cytokines is based on their diffusion diameter and the degradation/uptake mechanism of these cytokines in the interstitium. 


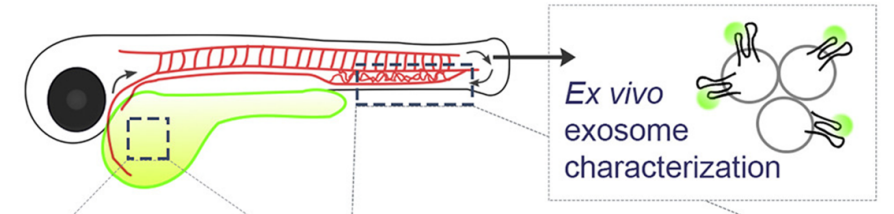

In vivo biogenesis \& distribution of endogenous exosomes

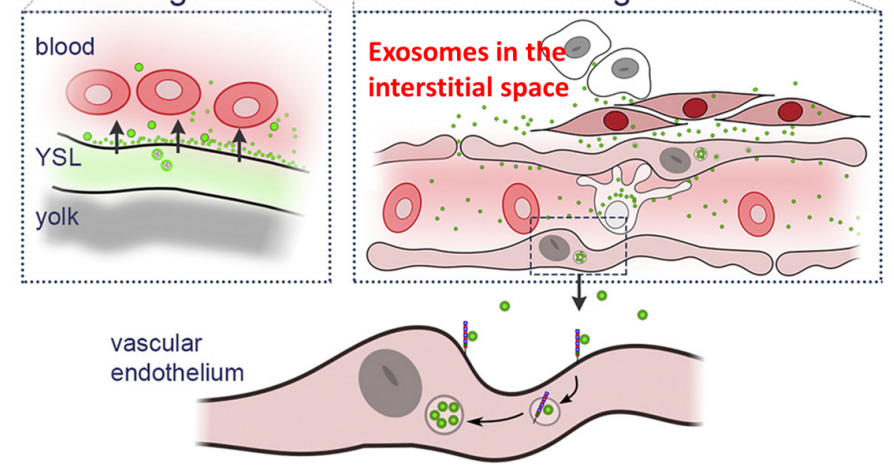

internalization pathway \& functional analysis

Figure 4. Live tracking of endogenous exosomes visualized the presence of numerous exosomes in the bloodstream but also in the interstitial space (reproduced with permission from reference 27). More detailed video clips can be found at https://doi.org/10.1016/j.devcel.2019.01.004.

We expect that exosomes act similarly to cytokines or paracrine mediators composed of peptides, lipids, lipopeptides or glycopeptides. This is an expansion of small molecules (several $\mathrm{nm}$ in diameter) and macromolecules (several to ten or tens of nanometers) to the extracellular vesicles (hundred to thousand nanometers). Interestingly, we call these extracellular vesicles as exosomes/ectosomes (around $100 \mathrm{~nm}$ ), microvesicles (up to $1 \mu \mathrm{m}$ ) and apoptotic bodies (larger than $1 \mu \mathrm{m})(1) .1$ gram of tissue $\left(10^{12} \mu \mathrm{m}^{3}\right)$ is considered to be packed by $10^{9}$ cells $\left(10^{3} \mu \mathrm{m}^{3}\right.$ per cell); however, if as sparse as one-tenth, $10^{8}$ cells might fill this 1-gram tissue and $0.1 \mathrm{~mL}$ of space $\left(10^{3} \mu \mathrm{m}^{3}\right)$ are open to being filled by macromolecules and endogenous nanomaterials called exosomes $\left(10^{2} \mathrm{~nm}^{3}\right)$. If as sparse as one-tenth, the remaining space can be filled by $10^{9}$ exosomes. In short, there is much room for exosomes to fill, to evacuate, or to traffic from the donor cells to recipient cells. Lymphatic flow has a very good driving force of transporting these exosomes from the donor cells to recipient cells. Even the brain was recently found to have inherent lymphatic flow(57-64) (Figure 5) and known to have such a variety of exosomes liberated by neurons, astrocytes, microglia and other cells.(65-69) Interstitial contents are cleared from the brain interstitium to the systemic circulation via neck lymph nodes both continuously and in circadian rhythmic fashion (70). Other tissues are all open to the lymphatics and thus lymphatics are to let the cellular and subcellular components (in this case, exosomes and microvesicles) to the systemic circulation. Lymphatic channels consisting of popliteal, inguinal, external iliac lymph nodes and brachial, axillary and subclavian lymph nodes are the well-known lymphatic drainage systems from lower and upper limbs, respectively. Internal iliac, celiac, mesenteric lymph nodes and channels, and azygous and hemiazygos lymphatics are from the pelvic and abdominal cavity. Lymphatic channels of lobal, hilar, and paratracheal lymph nodes are from the lungs. Head and neck have their own lymphatics and lymph nodes. And as the last revelation, we now know that even brain parenchyma has lymphatics as a drainage system.(57-64)

Most interesting was the brain lymphatics, (65-69) as brain lymphatics are heavily involved in clearing many abnormal proteins and protein aggregates.(71-73) Amyloid plaques and tau aggregates are well-known examples.(74-78) 


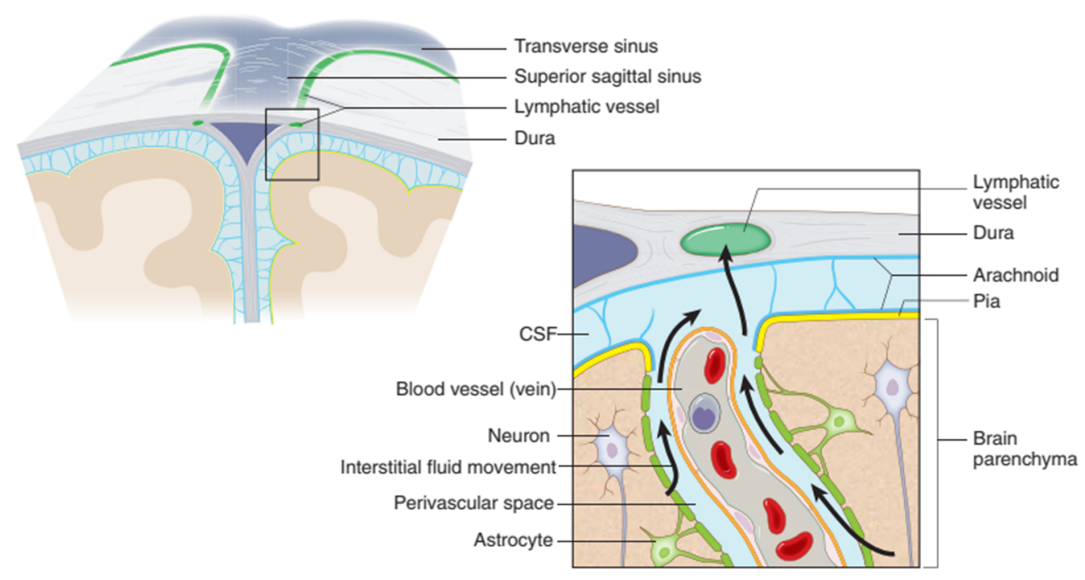

b

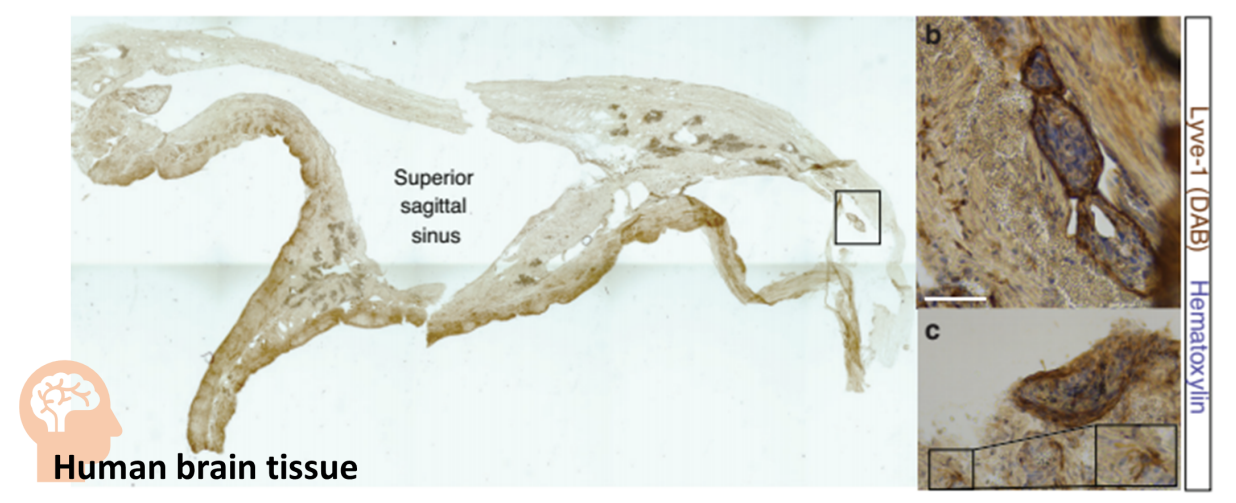

Figure 5. The lymphatic system in the brain. (a) A schematic illustration of the connection between the glymphatic system and the meningeal lymphatic system. (b) Lymphatic vessels identified in the human brain tissue. Reproduced with permission from reference 62 .

Tissue-resident macrophage, microglia are known to release many amounts of a diversity of exosomes.(78) The microglia release at least two types of exosomes, one of which help recovery from inflammation, the other of which exacerbate inflammation in situ of the brain. Microglia were supposed to do the dual roles in neuroinflammation in the traumatic brain or neurodegenerative diseases, too.

Interstitial exosomes are expected not to be consumed on-site and can be spilled out to the blood circulation. This is different from hormones composed of small molecules or small or large peptides, such as thyroxine, estrogen, testosterone, cortisol, or thyrotropin releasing hormone (TRH; 3 a.a.), gonadotrophin releasing hormone $(\mathrm{GnRH} ; 10$ a.a.), corticotrophin releasing hormone $(\mathrm{CRH}$; 41 a.a.), growth hormone releasing hormone (GHRH; 44 a.a.), adrenocorticotrophic hormone (ACTH; 39 a.a.), growth hormone (GH; 191 a.a.), prolactin (198 a.a.), thyroid stimulating hormone (TSH; 210 a.a.), follicle stimulating hormone (FSH; 207 a.a.), and luteinizing hormone (LH; 216 a.a.). All these hormones have direct access to the vascular system and even the portal venous system in case of GnRH, GHRH, TRH, and CRH. Exosomes should pass through cellular membranes of capillaries, at most two, i.e. abluminal and luminal. In this case, transcytosis is expected, however, in other cases, passage through the intercellular spaces can be expected.(79)

The size of exosomes and the package structure, whose contents are now considered to be customized to make specific types of exosomes for the delivery of their contents, make their passage through the basement membrane and either through inter-endothelial 
clefts in continuous endothelium of capillaries or fenestrated or sinusoid blood capillaries through pores. More likely, exosomes in the interstitial spaces can be transferred and drained through lymphatic vessels like the other materials in the interstitial fluids to the blood circulations. Here the most unique characteristics of lymphatic vessels are their one-way transfer, such as from the interstitial spaces to the inside of lymphatic vessels and no way back. The driving force is the pressure generated by interstitial fluid.

Systemic intravenous administration of exogenous exosomes might make these exosomes experience a unique situation that they are exposed to the blood flow immediately after administration and meet circulating cells and then reach and take rest in the sinusoids of the liver, bone marrow(80) and spleen.(81) Once passing through the pores of sinusoid capillaries of these organs, exosomes will have the chance to behave like endogenously produced exosomes. We need to estimate how much proportion of exosomes will reach the expected target tissues based on this understanding. Endogenously produced exosomes should be considered as the intercellular package-information messengers for paracrine action. And the circulatory reach of exosomes to the remote organs like hormones might be an infrequent phenomenon though in cases of inflammation, development or degeneration they might dominate. Thus, making systemically administered exosomes as therapeutic vehicles should be tried ingeniously or at least should be monitored using molecular imaging methods.

\section{Waste disposal of endogenous and exogenous exosomes}

Endogenous exosomes, if labeled transgenically, can be monitored for their biodistribution(27) (Figure 4). The recent investigation reported that exosome-marker labeled reporter gene could successfully label endogenous exosomes and this technology could visualize, in developing fish, the spillage of endogenous exosomes from the yolk to the circulation, their flow to reach the target, even the movement in the interstitial spaces or their clearance by circulating macrophages. Though this investigation was about the massive production and circulation of exosomes in developing embryo, it clearly showed the circulating endogenous exosomes, reaching venous plexus (capillary surrogates in fish) and interacting with only venous endothelium and not with arterial endothelium, and after reaching interstitial space, moving like Brownian movements confined to the space and not being taken up by cells in the interstitium including muscle cells. Circulating macrophages took up several of the exosomes but not the major player of clearance and instead endothelial cells took many exosomes. Similar findings were observed using melanoma cell line-derived exogenous exosomes administered to the blood circulation.(26) That is to say, injected exosomes circulated and reached endothelial cells and circulating macrophages. There have been few reports of exosomes reaching the interstitial space through the endothelial cells in the exogenous exosome model.

We can assume that the mass balance between production, delivery, clearance, and degradation influence the action at stationary states. Thus, the tumor-derived exogenous exosomes and embryo yolk-derived endogenous exosomes might have represented and visualized the status of high turn-over rates. $(26,27)$ Physiologic status of endogenous exosomes delivery and fate of uptakes were well visualized during embryo development and systemically administered exogenous exosomes showed similar behavior; however the delicate difference between these two imaging experiments was observed in that the number of exosomes seemed to determine their passage to interstitial space while circulating macrophages could capture only a small fraction of circulating exosomes. Exosome specificity in uptake looked like working.(27) and we need to be reminded that this real-time imaging visualizes only the small period after injection and the investigators did not yet pay attention to the clearance. 
a

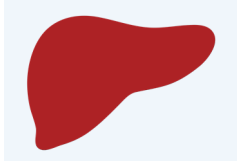

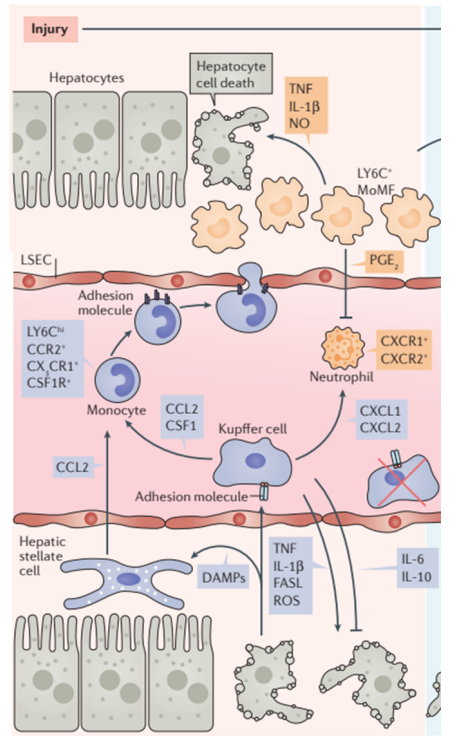

b

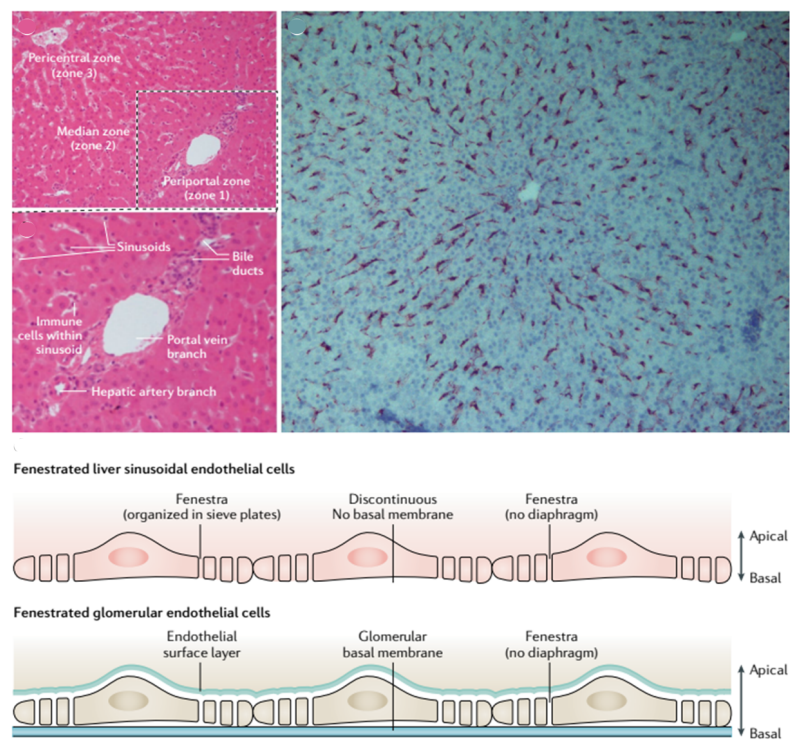

C

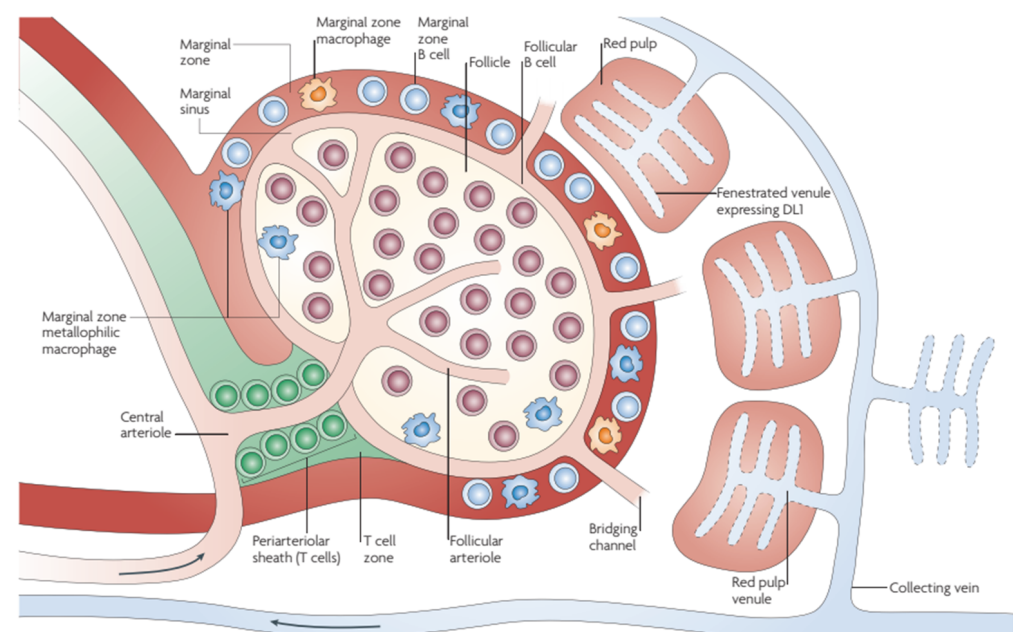

d

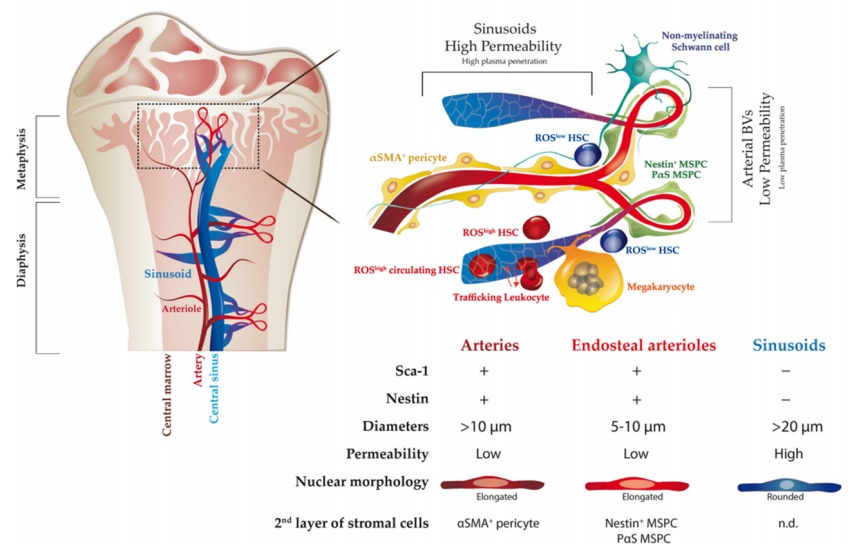

Figure 6. Waste disposal of endogenous and exogenous exosomes. (a) Kupffer cells in the acute liver injury aid the restoration by removing debris. In the same vein, exosomes may as well be removed by Kupffer cell (reproduced with permission from reference 86). (b-d) In the sinusoids of the liver, there is enough time for exosomes to interact with Kupffer cells, sinusoidal endothelial cells and hepatocytes, which makes it an important factor for clearance. Spleen and bone marrow also have sinusoidal structures. Macrophages and endothelial cells in this space may have more chance to take up exosomes and clear them. (reproduced with permission from reference 88, 90 and 80, respectively) 
Molecular imaging using fluorescent or radionuclide markers can elucidate the quantitative biodistribution of exogenous exosomes administered systemically.(1) Fluorescent marker-labeled exosomes can be used easily in transparent zebrafish; however, in small animals and humans, we need the imaging with the modality capable of repeated depth imaging enabling quantification. $(1,82)$ Bioluminescence imaging allows depth imaging in small animals without the capability of quantification but cannot be used for humans.(1, 83-85)

Tc-99m HMPAO, Tc-99m tin (RBC agents) or In-111 oxine are the agents for labeling exosomes and follow-up images of radionuclide labeled exosomes were acquired by several investigators.(1, 82) Interestingly, most of the systemically administered exogenous exosomes were shown to distribute to various organs different from each other in small animals. Most similar was that they showed liver uptake and itestinal activity.(33)

This might mean that hepatobiliary excretion would take place after the exosomes were taken up by hepatocytes. Later images had shown that liver activity decreased but remained. There is a possibility that liver uptake is partially by hepatocytes, and also liver-resident macrophages, such as Kupffer cells(86) (Figure 6A) and also endothelial cells of the liver sinusoids $(87,88)$ (Figure 6B). In the sinusoids of liver, spleen, and bone marrow, there are enough time of exosomes to interact with Kupffer cells, sinusoidal endothelial cells and hepatocytes. The burden of exosomes given to these cells and their specificity of interaction for uptake would determine the clearance of exosomes from the blood circulation.(89) Important is that the endothelial cells of the other peripheral tissues will compete with liver sinusoid endothelial cells. The flow speed might be different between lung capillary and periphery and liver sinusoids as well as the amount of interaction between exosomes and endothelial cells of these organs. And circulating mononuclear cells of monocytes/macrophages will also compete with Kupffer cells. In this competition, tissueresident macrophages will also benefit from the slow blood flow velocity in the liver.

The spleen has also the sinusoidal structure $(81,90)$ (Figure 6C) and bone marrow(80) (Figure 6D) too, but the former has a very harsh environment so that the exosomes would be under immune surveillance there and the latter would show more favorable milieu where marrow microenvironment supports the premature cells to become maturated to make blood cells.(91) Exosomes will have the chance to be taken up by endothelial cells of these organs of liver, spleen and bone marrow, however, most of the endocytosed exosomes experience the degradation within endosomelysosome compartments.

We suggest that the investigators need to seriously consider the sizable fraction of the exosomes for clearance from the living body and remainder is working to affect the target cells. This reminds that red blood cells are working with the designated period after production and release from the bone marrow with the lifetime of 120 days in humans and platelets much shorter time and granulocytes far shorter time with even several hours. Exosomes are not going to survive and take actions on targets throughout their lifetime but most of the excessive exosomes are to be cleared and recycled or excreted from the body. Many routes of excretion pathways might work in collaboration but in the sense of quantity, liver, and kidneys are the two major routes.

Most of the exosomes have specific targets just as enveloped viruses have target cells. Herpes, pox, hepatitis $\mathrm{B}$, corona, respiratory syncytial, influenza, yellow fever or dengue, and human immunodeficiency viruses are the examples. Every virus has been known to show tropism. Exosomes would behave similarly. By analogy, if the number of exosomes in the circulation are not enough to overcome the clearance capacity of the liver and other organs, no action will take place in the target organs or cells. This was the case with the molecular imaging results of radiolabeled exosomes, which showed dominant activity in the liver and other MPS organs. $(1,44,82)$ However, the paracrine action of the exosomes might work always, and the eloquent example is the exosomes of the brain (65-69). Unfortunately, the study of exosomes in the brain was not so fruitful to understand the significant roles of exosomes in the brain in diseases or in the physiologic setting. We reserved a separate section for explaining the importance of the 
roles of exosomes in the brain for its physiologic and disease-related status.

\section{Therapeutic effect follows the prerequisites} of uptake of exosomes

If exosomes have therapeutic efficacy, systemically administered exosomes should meet blood components and should not be considered seriously abnormal by the corona proteins of the plasma(92) and circulating mononuclear phagocytes.(27) Investigators examined corona protein wrapping of liposomes, and once engineered (produced in vitro), the exosomes should be considered as artificial, such as like liposomes, by immune cells. Thus, all the information regarding whereabouts of liposomes should be taken into consideration when we speculate the whereabouts of exosomes in vivo.(91-94)

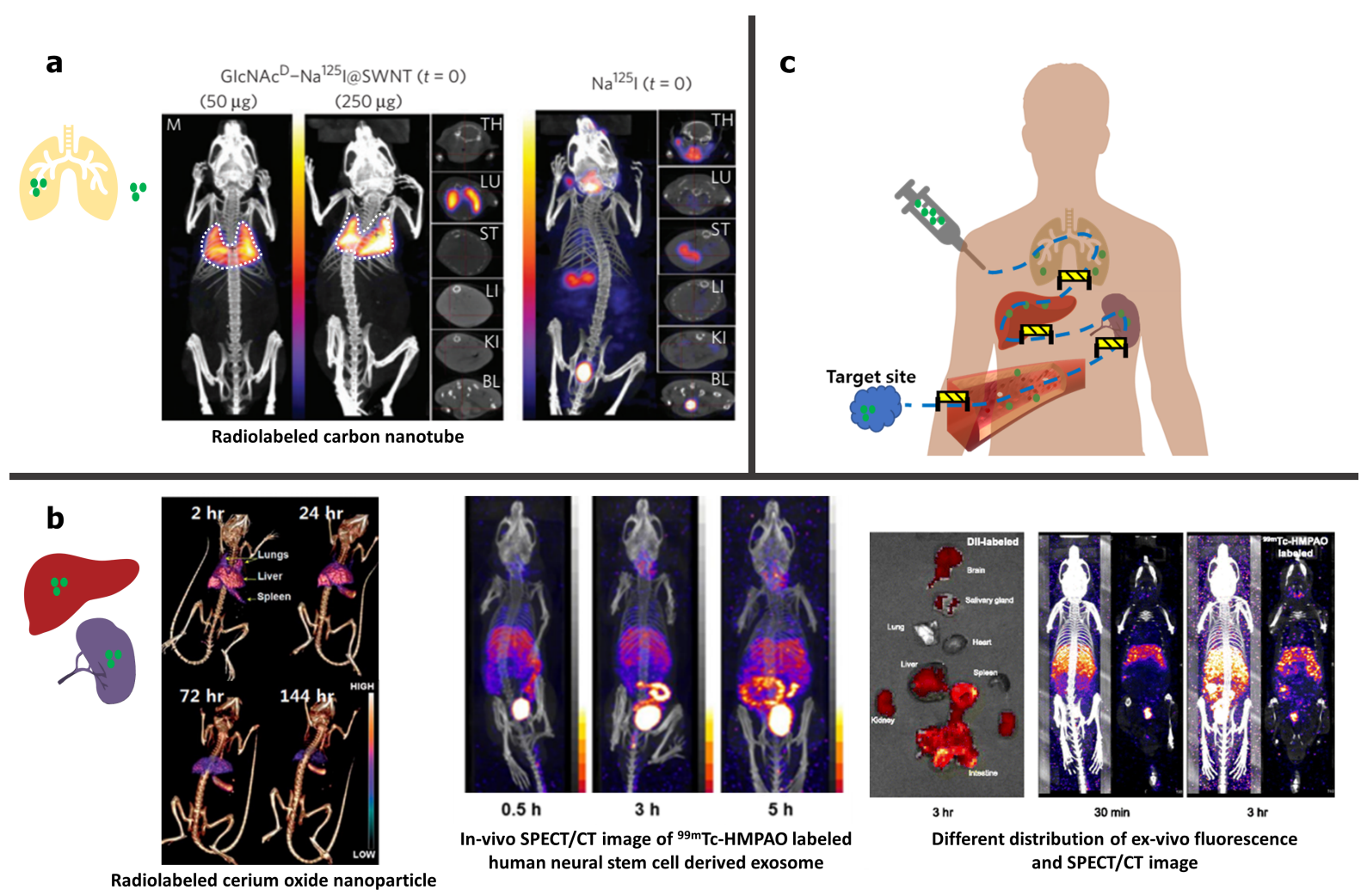

Figure 7. Inherent obstacles for targeted delivery of exosomes after systemic administration. (a) Whole-body SPECT/CT image after systemic administration of carbon nanotube labeled with radionuclide ${ }^{125}$ I showed a substantial amount of tracer accumulation in the lungs, reflecting immediate aggregation and filtration by alveolar capillaries (modified with permission from reference 95). (b) In vivo biodistribution of radiolabeled nanomaterials, showed accumulation in spleen and liver (131). Exosome radiolabeled with $99 \mathrm{mTc}-H M P A O$ showed similarly high accumulation in the spleen and liver, after systemic administration. At the delayed period in showed relatively high uptake along the intestine, reflecting the hepatobiliary excretion (yellow arrow). Interestingly, fluorescence and SPECT showed different distribution patterns, although simultaneously labeled. (modified with permission from reference 131, 29 and 24). (c) Systemically injected exosomes should avoid being captured in lungs, liver, and spleen, to reach the target site effectively.

This was important because we have a seriously flawed report of the earliest works to propose the plausibility of using carbon nanotubes that the injected carbon nanotubes would have aggregated immediately and were filtered by alveolar capillaries (95-98) (Figure 7A). In human lungs, 500 million alveoli are present and if 40 capillaries per alveolus are assumed, 20 billion capillaries are expected to be present in a healthy person. Lung perfusion scans of nuclear medicine are done with 100 thousand particles of Tc-99m macroaggregated albumin (MAA) particles of average $50 \mu \mathrm{m}$ of diameter. It blocks precapillary arterioles (capillary to precapillary arteriole ratio $=40$ ) and thus if we assume precapillary arteriole to alveolus ratio is one, MAA blocks less than one five-thousandth of alveolus-vascular units. Exosomes show inherently low colloidal stability, considering the zeta potential (from 
\pm 10 to \pm 30 ) (99). Furthermore, it is also reported that aggregation can occur in the production process of exogenous exosomes (100). Thus, exosomes will experience a similar fate once aggregated in vitro or in vivo after injection. Corona proteins, both hard and soft, might wrap up the exosomes but, no reports of such problems are in the literature. Only the failure of the carbon nanotube was easily found in the literature.(95-98)

Once exosomes escape from alveolar capillaries of the lungs after successful prevention from being taken up by alveolar capillaries, they are free to reach many organs, such that liver in $30 \%(7.5 \%$ arterial $22.5 \%$ venous flow $=$ intestinal $/$ stomach $/$ spleen blood flow) of systemic (non-pulmonary) blood flow (=cardiac output; C.O., left-sided), kidney in $20 \%$ of C.O., brain in $15 \%$ of C.O., spleen in $7.5 \%$ of C.O. and lungs in $100 \%$ of right-sided C.O. And thus, the other organs such as extremities contribute only $27.5 \%$. Though this summary ignored the variances, one can easily see the anatomical constraints of exosomes' distribution in the circulation system. In liver and spleen (responsible for $30 \%$ of C.O.) and their slow blood flow in their sinusoid system, exosomes have enough time for contacting the endothelial or parenchymal cell surfaces of the liver (Kupffer cells (Figure 7B) and endothelial cells) and spleen (red pulp macrophages and endothelial cells). In the lungs, all the exosomes shall meet pulmonary capillary endothelium which is continuous type like BBB, skeletal muscles, and skin but with a slow flow of through these capillaries. These provide a full chance of keeping in touch with endothelial cells.

Thus, to be effective, the exogenously delivered exosomes should reach the target tissues while escaping the captures by circulating macrophages, tissue-resident macrophages, especially Kupffer cells, splenic red pulp macrophages and alveolar macrophages and the endothelial cells of these three organs and hepatocytes (Figure 7C). These cell types have specific uptake mechanism. Among the variety of mechanism of exosomes' uptake in the recipient cells, phagocytosis might work when macrophages took exosomes or nonspecific pinocytosis in any professional or non-professional phagocytic cells, while endocytosis in the endothelial cells, either receptor-mediated clathrin-dependent or caveolin-dependent, might work in the endothelial cells in the capillaries of the lungs, liver, and spleen. There are also unknown mechanisms of uptake in hepatocytes. How much exosomes would reach the target tissues such as injured myocardium, brain, and tumors, can be assessed only by in vivo molecular imaging and should be interpreted using the above. Using nuclear molecular imaging, one can even quantify how much of the exogenous exosomes were taken up by these gatekeeper tissues such as lungs, liver, and spleen. $(44,51,82)$

Now, we can speculate that endogenous exosomes might experience the same clearance, uptake or phagocytosis, and degradation or little but existing possibility of recycling of endogenous exosomes in the lungs, liver, and spleen.(91) Also, the lymph nodes and other organs such as intestines and bone marrow also render the place of contact of exosomes with endothelial cells. Unlike sinusoid capillaries whose endothelium leak most of the particulate components to reach the underlying parenchymal tissues, intestines and renal glomeruli having fenestrated capillaries might challenge exosomes to pass through these fenestrae with a 60 to $80 \mathrm{~nm}$ diameter. However, intercellular clefts might be used for the exosomes to pass through the continuous capillaries of the muscles, brain, skin, and especially lungs to meet cells in the interstitial space.

Endogenous exosomes, if produced and passed through continuous (brain, muscle, skin, gonads) or fenestrated capillaries (endocrine glands, intestines) with massive amounts, most will be captured by lungs, liver and spleen and lymph nodes by tissue-resident macrophages and hepatocytes. The endocrine action of endogenous exosomes, if any, would not reach such a significant proportion as hormones and endocrine target tissues. The specificity of target tissues for specific types of exosomes was reported to exist in a few investigations(101) and is yet to be determined. There is a possibility that waste disposal prevails and then in a unique manner, the successfully escaped exosomes might work upon the target tissues in adult stationary individuals. We think it is highly likely that endocrine action of exosomes would take place 
when the deviation from the normal stationary states takes place in such cases as inflammation, injury, and degeneration and regeneration. Child growth or rapid aging might also be the ones of examples.

\section{Specificity of exosomes uptake and therapeutic effects}

Once in the extravascular spaces after systemic administration, exosomes might have therapeutic effects. To be able to render therapeutic effects, unlike hormones, exosomes might use receptor-mediated endocytosis (clathrin dependent), non-specific macropinocytosis or caveolae-dependent endocytosis and finally phagocytosis. $(17,22$,
102) Which types of recipient cells are using these mechanisms is not well delineated. If receptor-mediated endocytosis renders the specificity of the interaction between exosome and recipient cell, cell adhesion molecules will be involved (103-110). Among four groups of cell adhesion molecules (CAMs) of immunoglobulin (Ig) superfamily, cadherin, integrin and $\mathrm{C}$-type lectin-domain proteins (CTLD), integrins received attention as the first paper of organotropism reported that the formation of premetastatic niche was made possible by delivery of exosomes having $\alpha_{6} \beta_{4}$ (for laminin) and $\alpha_{6} \beta_{1}$ to the lungs and $\alpha_{v} \beta_{5}$ (for vitronectin) for the liver (111) (Figure 8A).

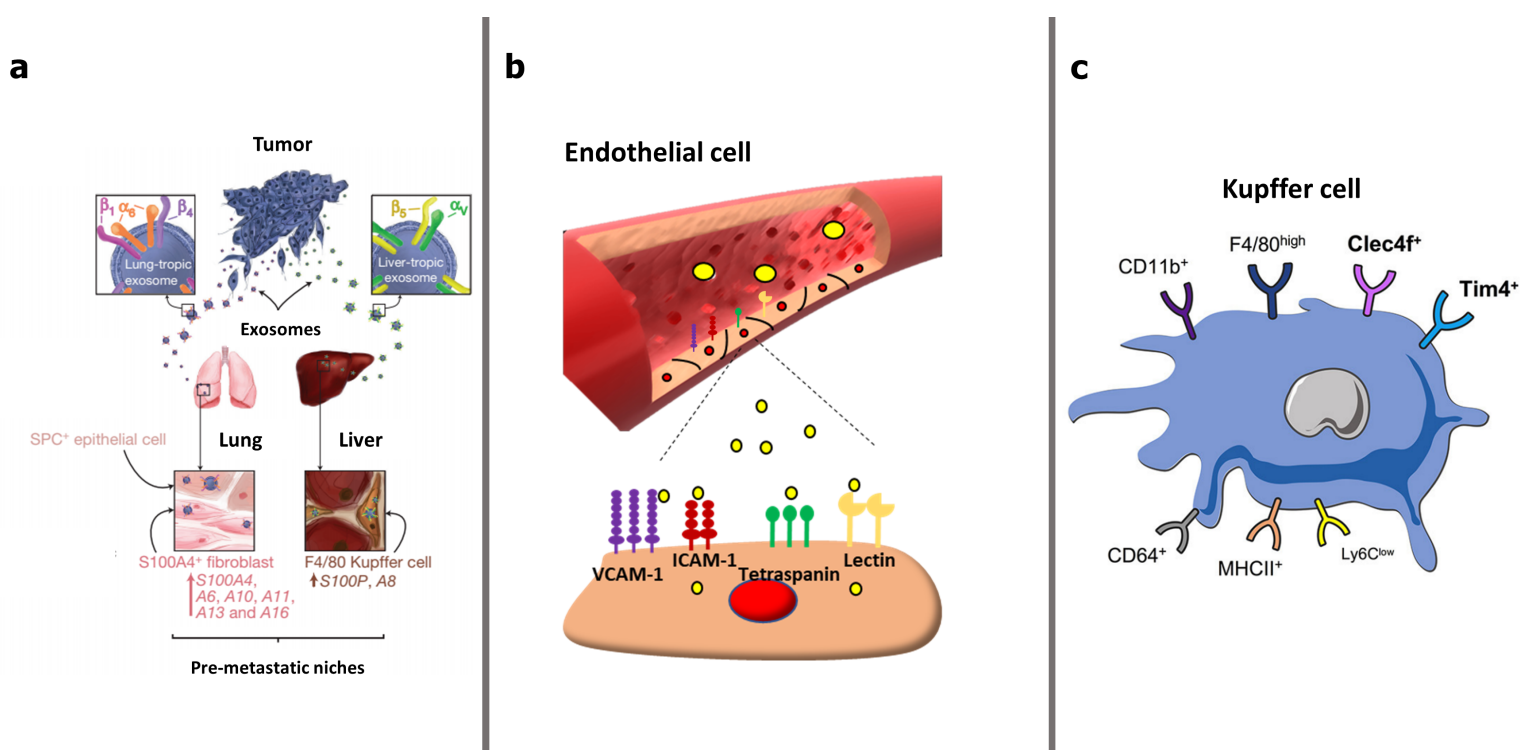

Figure 8. Specific uptake of exosomes. (a) Organotropic incorporation of tumor secreted exosomes via integrin-mediated organ-specific cell uptake (reproduced with permission from reference 111). (b) Cell surface proteins, which may have a role in receptor-mediated endocytosis of exosomes in the endothelial cells. (c) Surface receptors of Kupffer cell may specificallyuptake exosomes by recognizing Fc portion on exosome surface or recognizing exosomes as pathogens via pattern recognition. receptors (reproduced with permission from reference 112).

Among 24 integrins, proteomic analysis hinted the roles of exosomes having the above subtypes of integrins. ICAMs and VCAM are on the surface of endothelial cells (Figure 8B) and belong to Ig superfamily, and thus if exosomes use these CAM molecules, they will also use receptor-mediated endocytosis. In vivo zebrafish imaging experiments, exosomes stayed an average of 3 minutes on the surface of endothelial cells of the capillaries (venous plexus).(26, 27) Kupffer cells and probably the other tissue-resident macrophages use FcR receptors to find the client antibodies and if exosomes have Fc portion on the surface, they will be immediately recognized by Kupffer cells. However, there are more receptors on the surface of Kupffer cells, including CD14, Toll-like receptor 4 (TLR-4) and others (112).(Figure 8C) CD14 is the co-receptor of TLR4 and one of the pattern recognition receptors (PRR). If the exosomes are recognized via this receptor by the cells, exosomes can be said to be recognized like pathogens. 
The in vivo molecular imaging in zebrafish study impressively presented that venous endothelial cells solely contacted and took up them while arterial or other parenchymal cells including muscles beyond vessel walls ignored and did not take up the exosomes in the interstitial space.(27) In the previous reports in the literature, using just the in vitro system, there were vague reports that exosomes of mesenchymal stem cells were only taken up by mesenchymal stem cells(113) or that exosomes of cancer cells were taken up by cancer cells but not by fibroblasts.(19, 21) The opinions of cell specificity are so fragmented as they are based on scattered data acquired mostly in vitro. As was explained above, the discussion of these specificities should have included a diversity of cells including macrophages, endothelial cells, and even hepatocytes as probable targets. The specificity of exosomes' uptake in the recipient or bystander cells should be explained in terms of the differences in the mechanism of uptake in these different cells. Without insight about the mechanism, one cannot understand the differential uptake between cells of systemically administered exosomes.

Exogenous exosomes should be tested, in addition to their equivalence of the efficacy, for their similarity to the endogenous exosomes after being taken up by the recipient cells.(51-55) If investigators were to make chimeric(48) or surface modified exosomes,(49) they should prove that the modification did improve the capability of exosomes to avoid the uptake by macrophage/ endothelial cells or other parenchymal cells such as hepatocytes and thus increasing the enhanced uptake in the target cells. And then the contents of peptides, nucleic acids and even lipid mediators within exosomes will work in the early/late endosomes or finally endo-lysosomes.(102) We should comprehensively investigate to find out which kind of signaling molecules, modifiers or regulatory motifs or pathway components are working as therapeutic purposes, as differential effect investigations.

The specificity of production of endogenous exosomes

Cell biology has elucidated many details of the production of exosomes from the cells.(17,
18) The information is so concrete that we now differentiate the mechanism of production of vesicles; ectosomes using the mechanism of evagination of plasma membranes, exosomes using endosomal invagination with intraluminal vesicles in the multivesicular body, and microvesicles using similar evagination to ectosomes (17) (Figure 9A). Among these, multivesicular body and exosomal production were well characterized because parts of the process were in common with endocytosis and endolysosomal degradation pathways.(76) In fact, at first glance, the overlap is so delicate that one can miss the differential mechanism of their production for release and the degradation of exosomes after endocytosis.(17, 114-117) This mimicry, however, is enlightening in that the cells seemed to use a similar apparatus for degradation and intercellular communication.

Biogenesis of exosomes is now known to run with the ESCRT-dependent pathway and nonESCRT-dependent pathway $(18,114,118)$ (Figure 9B). Intraluminal vesicles can be associated with the pre-arranged excretion, but also go through lysosomal or autophagosomal association to be degraded $(114,117)$ (Figure 9C). So far, how the cells choose the production pathway between ESCRT-dependent or independent ones and how the exosomes' membranes or exosomes' intraluminal contents are packaged are not well understood. Obviously, the membrane contents shall include the membrane contents of the cells and species or individual identity (as in $\mathrm{MHCl}$ or MHC2) as surface-exposed molecules. Undoubtedly, the mechanism of recognition of the exosomes by the recipient cells depends upon the surface characteristics of exosomes. $(17,22,102,107)$

While we think about the diversity of receptors of the recipient cells, such as Ig superfamily, endocytosis-prone receptors for ligands, scavenger receptor, peptidoglycan or sphingomyelins and the similar, positioning the surface molecules inappropriate amount at correct loci along the membranes would be important. If bioengineering is planned, investigators need to characterize the factors and then modify the relevant molecules by inserting, deleting, or removing them. Of course, the consequences of the engineered modification of exosomes' surfaces should be 
evaluated for their impact 1) on the biogenesis (and especially packaging), 2) mechanism to determine whether to release them, 3) avoidance of clearance apparatus of endothelial cells, macrophages and hepatocytes or other parenchymal cells in vivo and 4) target cells' willingness to take up those exosomes in vivo. This is true for exosomes for their expected endocrine (or systemic) action, and especially when the major action mechanism of exosomes was known or expected to be for paracrine activities. There should be an obstacle to understand/overcome if investigators are trying to make inherently paracrine-major exosomes work as endocrine-functioning exosomes. They need to overcome the difference of milieu of paracrine and endocrine action while the harsh intravascular flow and the surveillance by patrolling and tissue-resident macrophages and ubiquitous endothelial cells will surely be a great hurdle.

Feasibility of using therapeutic exosomes for brain diseases

Therapeutic use of exosomes for brain diseases depends upon unique issues of the brain such as (a) passage of exosomes through the $\mathrm{BBB},(4, \quad 6,16,47,79,119) \quad(\mathrm{b})$ understanding the roles of exosomes as paracrine actors for intercellular communications, $(7,12,24,65,66,69,75,78)$ and 3) incomplete understanding of the cells involved in the production and uptake of exosomes. $(17,67,68)$ Systemic administration and waiting for the therapeutic effect were the prevalent strategy used by many investigators, however, the spatiotemporal nature of the dynamic complexity of exosomes production and uptake makes the effect unpredictable.

Uniquely, brain cells and most the neurons are doing different roles in different areas of the brain and there are found a diversity of brain cells in the same region meaning astrocytes, microglia, oligodendrocytes and other parenchymal cells in addition to a variety of neurons. Almost all the cells release exosomes and all the other cells take up exosomes. $(65,67-$ $69,120)$ There have been not many investigations matching these interactions and summary was tried in a fine review a while ago (67) (Figure 10A). The recipient cells would have specific receptors such as neural CAM (NCAM), adherin, selectin and immunoglobulin superfamily (IgSF), intercellular adhesion molecule (ICAM) and yet-to-be found many adhesion molecules.(68, 101, 107)

Neurotransmitters of small molecules or small peptides transmit the information between neurons and astrocytes. Synaptic cleft and neuron-astrocyte couples have been keeping and exchanging these transmitters around the close vicinity. However, in the brain as well as in other organs, there is extracellular space and once the brain cells are releasing exosomes for intercellular communication, this space also called interstitium is filled with exosomes anytime anywhere.(67) Paracrine action of exosomes will take place in interstitial space and the recipient cells are going to receive selective signals.(27) The easiest one to understand might be the message in the exosomes released by microglia. This has been called inflammation. However, there are already dual roles of microglial exosomes, one is for inflammation leading to tissue injury and the other is for the contribution of regeneration.(78)

If we imagine the situation that the brain is about to exchange its contents with the outside of the brain, one is the blood and the other is lymphatics. Recently, the existence of lymphatics in the brain was elucidated $(59,62)$. Blood to brain transport is controlled by $\mathrm{BBB}$ surrounding brain and BBB is made of a capillary with continuous endothelium. $(4,79)$ This means that materials should pass through the tight margins of cells from blood to brain interstitium. Once the cells in the brain release any material such as popular neurotransmitters or recently characterized exosomes, the fate of small molecules will depend on mainly the electrical, chemical, structure-functional, hydrophobicity/hydrophilicity characteristics.(79) The fate of released exosomes is to be determined similarly to small molecules but the factors for exosomes might be firstly the size, and then the surface characteristics determined by membrane peptides (such as ligands) and lipids (such as phosphatidylserine). Lipid bilayer structure of exosomes, considering their lipophilicity, can pass through the continuous membranes.(58-63) This is the basic wishful belief of the investigators who are doing research on using therapeutic exosomes for brain diseases. 
a

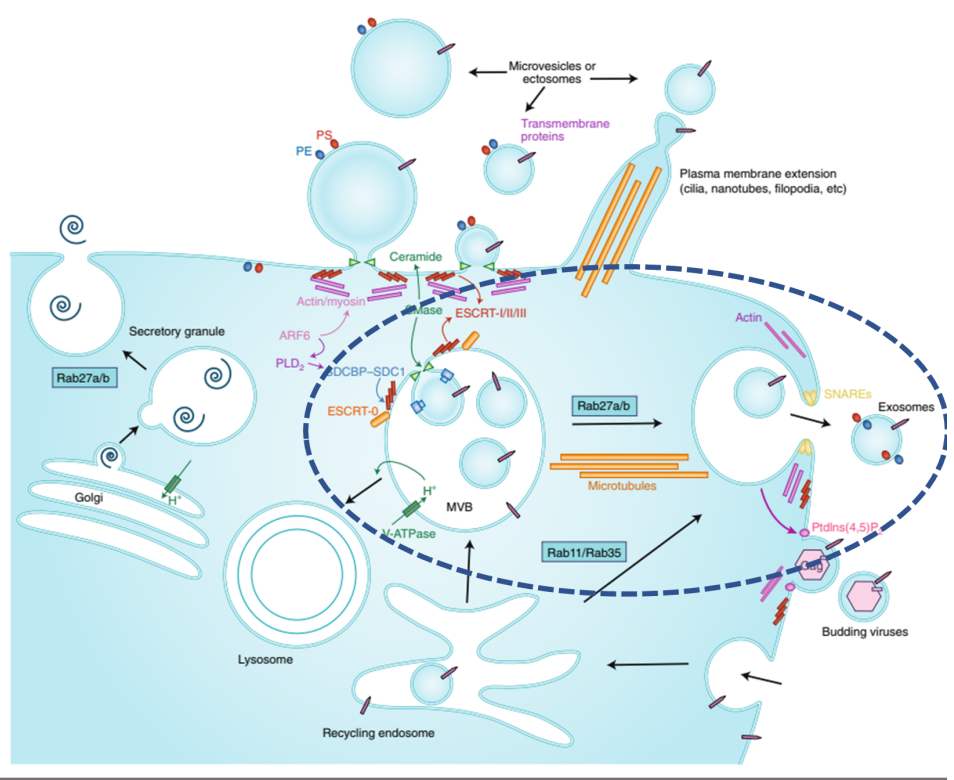

b

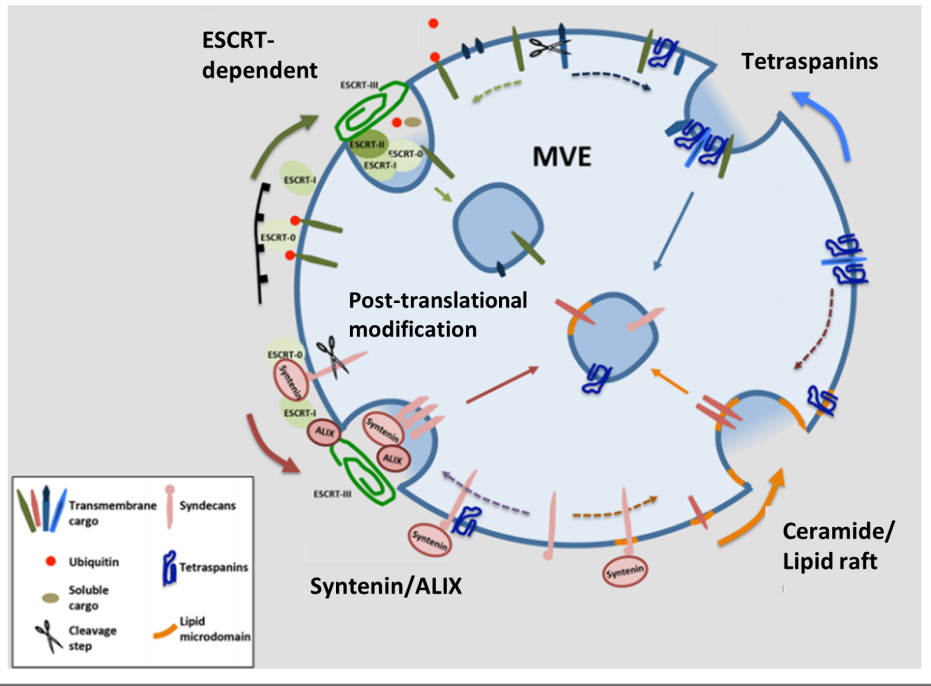

C

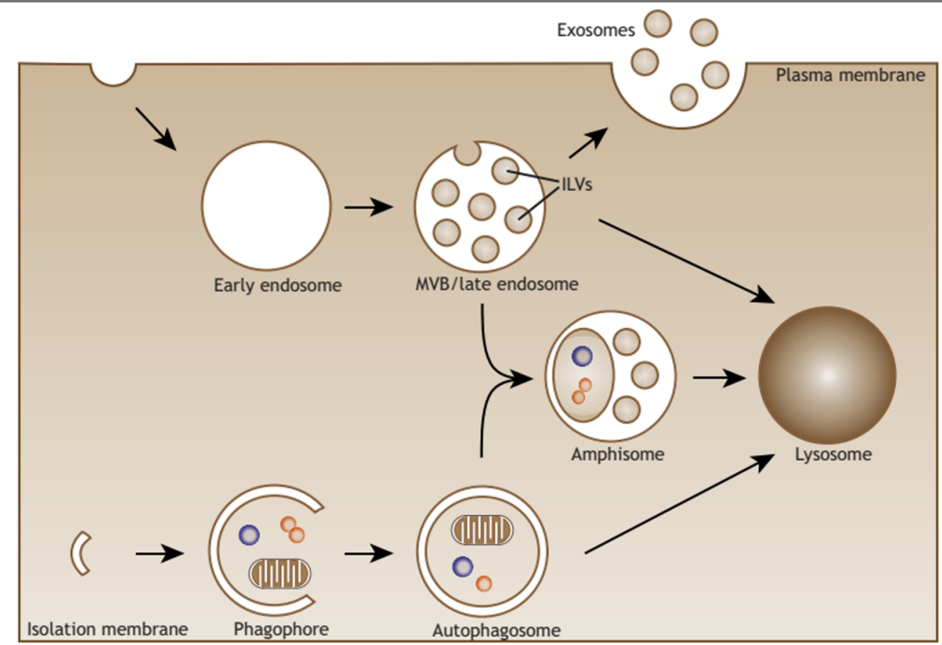

Figure 9. The specific production of endogenous exosomes. (a) Intracellular molecular pathways involved in the production and secretion of exosomes in late endosomal multivesicular bodies (modified with permission from reference 1). (b) The sorting mechanism, including both ESCRT-dependent and non-ESCRT-dependent pathways, at multivesicular endosomes involved in exosome production (modified with permission from reference 2). (c) Summary of classic endocytic pathway, autophagy and exosome production (reproduced with permission from reference 117). 
In fact, exosomes were taken up easily by the recipient cells on microfluidic chip study.(121) We could easily show that the same type of cells is taking up the exosomes released by the same cells.(49, 108, 113, 121) Furthermore, differentiated cells from progenitors also took up exosomes from the progenitor cells. The combination of cell study is pending; however, these experiments were all in vitro. Brain lymphatics associated with glymphatic $(58,70$, 122) were found to clear extra amount of materials produced on-site in the brain as well as materials transported in from the blood (Figure 10B).

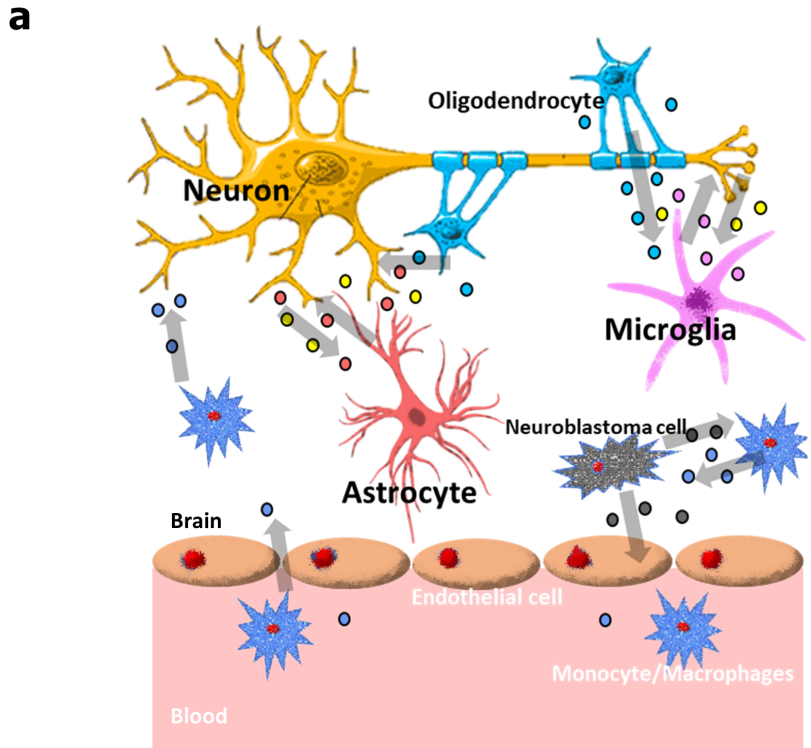

b

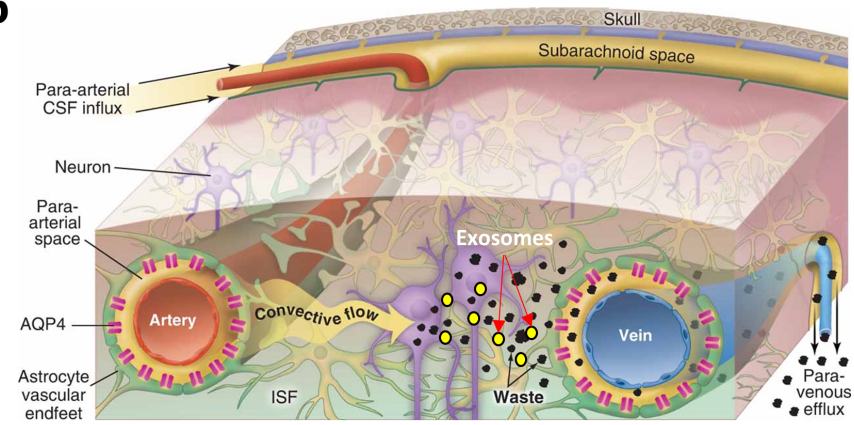

Figure 10. Exosomes in the brain. (a) Summary of exosomes in intercellular communication involving brain cells. (b) Conceptual figure indicating clearance of exosomes through glymphatic (modified with permission from Reference 122).

These materials include exosomes, and if exosomes carry modified abnormal proteins such as abnormal tau( $23,73,123)$ or alphasynuclein, $(24,74,124)$ they could be the vehicle of clearance of those abnormal proteins. Even extracellular amyloid could be cleared without or within exosomes. $(62,75,76,78$, 120) The mechanism of how those abnormal proteins are packaged in exosomes, are to be elucidated.(18, 67, 114, 120, 125) To predict the possible therapeutic roles of exosomes, we need to understand the paracrine action of exosomes and the clearance mechanism of exosomes' contents as well as the roles of professional phagocytic cells, microglia for this endeavor or non-professional phagocytic cells, if any in the brain.

To find out the feasibility of therapeutic use of exosomes by systemic administration, in vivo confirmation of therapeutic reach to the targets should follow. And for this, imaging with radionuclide-labeled exosomes might be a nextstep.(126-129) This is an in vivo companion diagnostic for promising therapeutic exosomes or simply expressed 'in vivo theranostics'. Fortunately, in vivo, well-established animal models of peptide propagation(130) or neuroinflammation are there while rendering a good opportunity for the investigators to predict the feasibility of using therapeutic exosomes for neurodegenerative diseases. 


\section{Conclusion}

There has been growing interest in the field of exosomes research. Based on successful results from in vitro and several in vivo studies, we have drawn a hopeful future of exosomes as a therapeutic agent. However, there is a significant gap between the expected therapeutic effect of exosomes and the actual clinical application. In order to fill this gap, a thorough understanding of exosomes is necessary, including the physiology of endogenous exosomes, distribution, and excretion after systemic administration and specific production.

We must realize that:

- Exosomes have a unique function in intercellular signaling and waste management. The mass balance between production, delivery, clearance, and degradation influence this function.

- Intercellular signaling of endogenous exosomes can take place in both a paracrine and endocrine manner. Their paracrine effect seems to be the dominant action in intercellular signaling, while the endocrine effect if any, must pass through the adversity of circulation and disposal system.

- Exogenous exosomes, when systemically injected, face many hurdles, such as professional or non-professional phagocytic cells, especially in sinusoidal spaces of specific organs.

- After reaching the target, exosomes are specifically taken up by the recipient cells. However, it can also be taken up by various bystander cells, thus differential uptake mechanisms in various cells should be elucidated.

- Understanding of BBB, paracrine action and glymphatic are essential to evaluate the feasibility of using exosomes as therapeutic agents for brain diseases.

- In vivo molecular imaging is essential to ensure that exosomes pass through the hurdles and reach the target, and to track the clearance.

For the clinical application of exosomes, we will find a way. We always have.

The authors declare no conflicts of interest. For signed statements, please contact the journal office: editor@precisionnanomedicine.com

Quote this article as Lee DS, Suh M, Kang SY, Hwang DW, Physiologic constraints of using exosomes in vivo as systemic delivery vehicles, Precis. Nanomed. 2019;2(3):344-369, https://doi.org/10.33218/prnano2(3)070819.1

\section{References}

1. Choi H, Lee DS. Illuminating the physiology of extracellular vesicles. Stem Cell Res Ther. 2016;7(1):55.

2. Choi H, Lee DS. Endogenous radionanomedicine: Biodistribution and imaging. Lee DS, editor: Springer; 2018.

3. Wiklander OP, Nordin JZ, O'Loughlin A, Gustafsson Y, Corso G, Mager I, et al. Extracellular vesicle in vivo biodistribution is determined by cell source, route of administration and targeting. J Extracell Vesicles. 2015;4:26316.

4. Chen CC, Liu L, Ma F, Wong CW, Guo XE, Chacko JV, et al. Elucidation of exosome migration across the blood-brain barrier model in vitro. Cell Mol Bioeng. 2016;9(4):509-29.

5. Das CK, Jena BC, Banerjee I, Das S, Parekh A, Bhutia SK, et al. Exosome as a novel shuttle for delivery of therapeutics across biological barriers. Mol Pharm. 2019;16(1):24-40.

6. Gu X, Chen H, Gao X. Nanotherapeutic strategies for the treatment of Alzheimer's disease. Ther Deliv. 2015;6(2):177-95.

7. Holm MM, Kaiser J, Schwab ME. Extracellular vesicles: Multimodal envoys in neural maintenance and repair. Trends Neurosci. 2018;41(6):360-72.

8. Kamerkar S, LeBleu VS, Sugimoto H, Yang S, Ruivo CF, Melo SA, et al. Exosomes facilitate therapeutic targeting of oncogenic KRAS in pancreatic cancer. Nature. 2017;546(7659):498-503. 
9. Kibria G, Ramos EK, Wan Y, Gius DR, Liu H. Exosomes as a drug delivery system in cancer therapy: Potential and challenges. Mol Pharm. 2018;15(9):3625-33.

10. Koh E, Lee EJ, Nam GH, Hong Y, Cho E, Yang Y, et al. Exosome-SIRPalpha, a CD47 blockade increases cancer cell phagocytosis. Biomaterials. 2017;121:121-9.

11. Qu M, Lin Q, Huang L, Fu Y, Wang L, He S, et al. Dopamine-loaded blood exosomes targeted to brain for better treatment of Parkinson's disease. J Control Release. 2018;287:156-66.

14. Yim N, Ryu SW, Choi K, Lee KR, Lee S, Choi H, et al. Exosome engineering for efficient intracellular delivery of soluble proteins using optically reversible protein-protein interaction module. Nat Commun. 2016;7:12277.

15. Yuan D, Zhao Y, Banks WA, Bullock KM, Haney M, Batrakova E, et al. Macrophage exosomes as natural nanocarriers for protein delivery to inflamed brain. Biomaterials. 2017;142:1-12.

12. Smyth T, Kullberg M, Malik N, Smith-Jones P, Graner MW, Anchordoquy TJ. Biodistribution and delivery efficiency of unmodified tumor-derived exosomes. J Control Release. 2015;199:145-55.

13. Takahashi Y, Nishikawa M, Shinotsuka H, Matsui Y, Ohara S, Imai T, et al. Visualization and in vivo tracking of the exosomes of murine melanoma B16-BL6 cells in mice after intravenous injection. J Biotechnol. 2013;165(2):77-84.

16. Zheng M, Huang M, Ma X, Chen H, Gao X. Harnessing exosomes for the development of brain drug delivery systems. Bioconjug Chem. 2019;30(4):994-1005.

17. Mathieu M, Martin-Jaular L, Lavieu G, Thery C. Specificities of secretion and uptake of exosomes and other extracellular vesicles for cell-to-cell communication. Nat Cell Biol. 2019;21(1):9-17.

18. Palmulli R, van Niel G. To be or not to be... secreted as exosomes, a balance finely tuned by the mechanisms of biogenesis. Essays Biochem. 2018;62(2):177-91.

19. Kao CY, Papoutsakis ET. Extracellular vesicles: exosomes, microparticles, their parts, and their targets to enable their biomanufacturing and clinical applications. Curr Opin Biotechnol. 2019;60:89-98.

20. Costa Verdera H, Gitz-Francois JJ, Schiffelers RM, Vader P. Cellular uptake of extracellular vesicles is mediated by clathrin-independent endocytosis and macropinocytosis. J Control Release. 2017;266:100-8.

21. Cantaluppi V, Gatti S, Medica D, Figliolini F, Bruno S, Deregibus MC, et al. Microvesicles derived from endothelial progenitor cells protect the kidney from ischemia-reperfusion injury by microRNAdependent reprogramming of resident renal cells. Kidney Int. 2012;82(4):412-27.

22. Horibe S, Tanahashi T, Kawauchi S, Murakami Y, Rikitake Y. Mechanism of recipient celldependent differences in exosome uptake. BMC Cancer. 2018;18(1):47.

23. Asai H, Ikezu S, Tsunoda S, Medalla M, Luebke J, Haydar T, et al. Depletion of microglia and inhibition of exosome synthesis halt tau propagation. Nat Neurosci. 2015;18(11):1584-93.

24. Xia Y, Zhang G, Han C, Ma K, Guo X, Wan F, et al. Microglia as modulators of exosomal alphasynuclein transmission. Cell Death Dis. 2019;10(3):174.

25. Perets N, Betzer O, Shapira R, Brenstein S, Angel A, Sadan T, et al. Golden exosomes selectively target brain pathologies in neurodegenerative and neurodevelopmental disorders. Nano Lett. 2019.

26. Hyenne V, Ghoroghi S, Collot M, Bons J, Follain G, Harlepp S, et al. Studying the fate of tumor extracellular vesicles at high spatiotemporal resolution using the zebrafish embryo. Dev Cell. 2019;48(4):554-72 e7.

27. Verweij FJ, Revenu C, Arras G, Dingli F, Loew D, Pegtel DM, et al. Live tracking of inter-organ communication by endogenous exosomes in vivo. Dev Cell. 2019;48(4):573-89 e4.

28. Lai CP, Kim EY, Badr CE, Weissleder R, Mempel TR, Tannous BA, et al. Visualization and tracking of tumour extracellular vesicle delivery and RNA translation using multiplexed reporters. Nat Commun. 2015;6:7029.

29. Arrighetti N, Corbo C, Evangelopoulos M, Pasto A, Zuco V, Tasciotti E. Exosome-like nanovectors for drug delivery in cancer. Curr Med Chem. 2018.

30. Corbo C, Cromer WE, Molinaro R, Toledano Furman NE, Hartman KA, De Rosa E, et al. Engineered biomimetic nanovesicles show intrinsic anti-inflammatory properties for the treatment of inflammatory bowel diseases. Nanoscale. 2017;9(38):14581-91. 
31. Molinaro R, Evangelopoulos M, Hoffman JR, Corbo C, Taraballi F, Martinez JO, et al. Design and development of biomimetic nanovesicles using a microfluidic approach. Adv Mater. 2018;30(15):e1702749.

32. Lee DS, Shin YK. Innate immunity to nanomaterials. Lee DS, editor: Springers; 2018.

33. Lee DS, Im HJ, Lee YS. Radionanomedicine: widened perspectives of molecular theragnosis. Nanomedicine. 2015;11(4):795-810.

34. Ha S, Lee DS. Endogenous radionanomedicine: Validation of therapeutic potential. Lee DS, editor: Springer; 2018.

35. Kraynov E, Kamath AV, Walles M, Tarcsa E, Deslandes A, Iyer RA, et al. Current approaches for absorption, distribution, metabolism, and excretion characterization of antibody-drug conjugates: An industry white paper. Drug Metab Dispos. 2016;44(5):617-23.

36. Mandai M, Kurimoto Y, Takahashi M. Autologous induced stem-cell-derived retinal cells for macular degeneration. N Engl J Med. 2017;377(8):792-3.

37. McGill TJ, Stoddard J, Renner LM, Messaoudi I, Bharti K, Mitalipov S, et al. Allogeneic iPSCderived RPE cell graft failure following transplantation into the subretinal space in nonhuman primates. Invest Ophthalmol Vis Sci. 2018;59(3):1374-83.

38. Gomez-Mantilla JD, Troconiz IF, Parra-Guillen Z, Garrido MJ. Review on modeling anti-antibody responses to monoclonal antibodies. J Pharmacokinet Pharmacodyn. 2014;41(5):523-36.

39. Kuriakose A, Chirmule N, Nair P. Immunogenicity of biotherapeutics: Causes and association with posttranslational modifications. J Immunol Res. 2016;2016:1298473.

40. Boswell CA, Mundo EE, Zhang C, Bumbaca D, Valle NR, Kozak KR, et al. Impact of drug conjugation on pharmacokinetics and tissue distribution of anti-STEAP1 antibody-drug conjugates in rats. Bioconjug Chem. 2011;22(10):1994-2004.

41. Shen BQ, Xu K, Liu L, Raab H, Bhakta S, Kenrick M, et al. Conjugation site modulates the in vivo stability and therapeutic activity of antibody-drug conjugates. Nat Biotechnol. 2012;30(2):184-9.

42. Arandjelovic S, Ravichandran KS. Phagocytosis of apoptotic cells in homeostasis. Nat Immunol. 2015;16(9):907-17.

43. Im HJ. Factors affecting biodistribution and their consequences: Excretion and Clearance. Lee DS, editor: Springer; 2018.

44. Seo HJ, Nam SH, Im HJ, Park JY, Lee JY, Yoo B, et al. Rapid hepatobiliary excretion of micelleencapsulated/radiolabeled upconverting nanoparticles as an integrated form. Sci Rep. 2015;5:15685.

45. Lai RC, Yeo RW, Tan KH, Lim SK. Mesenchymal stem cell exosome ameliorates reperfusion injury through proteomic complementation. Regen Med. 2013;8(2):197-209.

46. Vandergriff A, Huang K, Shen D, Hu S, Hensley MT, Caranasos TG, et al. Targeting regenerative exosomes to myocardial infarction using cardiac homing peptide. Theranostics. 2018;8(7):1869-78.

47. Alvarez-Erviti L, Seow Y, Yin H, Betts C, Lakhal S, Wood MJ. Delivery of siRNA to the mouse brain by systemic injection of targeted exosomes. Nat Biotechnol. 2011;29(4):341-5.

48. Zhang KL, Wang YJ, Sun J, Zhou J, Xing C, Huang G, et al. Artificial chimeric exosomes for antiphagocytosis and targeted cancer therapy. Chem Sci. 2019;10(5):1555-61.

49. Charoenviriyakul C, Takahashi Y, Morishita M, Nishikawa M, Takakura Y. Role of extracellular vesicle surface proteins in the pharmacokinetics of extracellular vesicles. Mol Pharm. 2018;15(3):1073-80.

50. Zhu Q, Heon M, Zhao Z, He M. Microfluidic engineering of exosomes: editing cellular messages for precision therapeutics. Lab Chip. 2018;18(12):1690-703.

51. Hwang DW, Choi H, Jang SC, Yoo MY, Park JY, Choi NE, et al. Noninvasive imaging of radiolabeled exosome-mimetic nanovesicle using (99m)Tc-HMPAO. Sci Rep. 2015;5:15636.

52. Jang SC, Kim OY, Yoon CM, Choi DS, Roh TY, Park J, et al. Bioinspired exosome-mimetic nanovesicles for targeted delivery of chemotherapeutics to malignant tumors. ACS Nano. 2013;7(9):7698710 .

53. Jo W, Jeong D, Kim J, Cho S, Jang SC, Han C, et al. Microfluidic fabrication of cell-derived nanovesicles as endogenous RNA carriers. Lab Chip. 2014;14(7):1261-9.

54. Jo W, Kim J, Yoon J, Jeong D, Cho S, Jeong H, et al. Large-scale generation of cell-derived nanovesicles. Nanoscale. 2014;6(20):12056-64. 
55. Oh K, Kim SR, Kim DK, Seo MW, Lee C, Lee HM, et al. In vivo differentiation of therapeutic insulin-producing cells from bone marrow cells via extracellular vesicle-mimetic nanovesicles. ACS Nano. 2015;9(12):11718-27.

56. Chao MP, Weissman IL, Majeti R. The CD47-SIRPalpha pathway in cancer immune evasion and potential therapeutic implications. Curr Opin Immunol. 2012;24(2):225-32.

57. Absinta M, Ha SK, Nair G, Sati P, Luciano NJ, Palisoc M, et al. Human and nonhuman primate meninges harbor lymphatic vessels that can be visualized noninvasively by MRI. Elife. 2017;6.

58. Louveau A, Plog BA, Antila S, Alitalo K, Nedergaard M, Kipnis J. Understanding the functions and relationships of the glymphatic system and meningeal lymphatics. J Clin Invest. 2017;127(9):3210-9.

59. Aspelund A, Antila S, Proulx ST, Karlsen TV, Karaman S, Detmar M, et al. A dural lymphatic vascular system that drains brain interstitial fluid and macromolecules. J Exp Med. 2015;212(7):991-9.

60. Da Mesquita S, Louveau A, Vaccari A, Smirnov I, Cornelison RC, Kingsmore KM, et al. Functional aspects of meningeal lymphatics in ageing and Alzheimer's disease. Nature. 2018;560(7717):185-91.

61. Louveau A, Da Mesquita S, Kipnis J. Lymphatics in neurological disorders: A neuro-lymphovascular component of multiple sclerosis and Alzheimer's disease? Neuron. 2016;91(5):957-73.

62. Louveau A, Smirnov I, Keyes TJ, Eccles JD, Rouhani SJ, Peske JD, et al. Structural and functional features of central nervous system lymphatic vessels. Nature. 2015;523(7560):337-41.

63. Louveau A, Herz J, Alme MN, Salvador AF, Dong MQ, Viar KE, et al. CNS lymphatic drainage and neuroinflammation are regulated by meningeal lymphatic vasculature. Nat Neurosci. 2018;21(10):138091.

64. Antila S, Karaman S, Nurmi H, Airavaara M, Voutilainen MH, Mathivet T, et al. Development and plasticity of meningeal lymphatic vessels. J Exp Med. 2017;214(12):3645-67.

65. Bahrini I, Song JH, Diez D, Hanayama R. Neuronal exosomes facilitate synaptic pruning by upregulating complement factors in microglia. Sci Rep. 2015;5:7989.

66. Glebov K, Lochner M, Jabs R, Lau T, Merkel O, Schloss P, et al. Serotonin stimulates secretion of exosomes from microglia cells. Glia. 2015;63(4):626-34.

67. Janas AM, Sapon K, Janas T, Stowell MH, Janas T. Exosomes and other extracellular vesicles in neural cells and neurodegenerative diseases. Biochim Biophys Acta. 2016;1858(6):1139-51.

68. Mulcahy LA, Pink RC, Carter DR. Routes and mechanisms of extracellular vesicle uptake. J Extracell Vesicles. 2014;3.

69. Wang S, Cesca F, Loers G, Schweizer M, Buck F, Benfenati F, et al. Synapsin I is an oligomannosecarrying glycoprotein, acts as an oligomannose-binding lectin, and promotes neurite outgrowth and neuronal survival when released via glia-derived exosomes. J Neurosci. 2011;31(20):7275-90.

70. Plog BA, Nedergaard M. The glymphatic system in central nervous system health and disease: Past, present, and future. Annu Rev Pathol. 2018;13:379-94.

71. Jessen NA, Munk AS, Lundgaard I, Nedergaard M. The glymphatic system: A beginner's guide. Neurochem Res. 2015;40(12):2583-99.

72. Rasmussen MK, Mestre H, Nedergaard M. The glymphatic pathway in neurological disorders. Lancet Neurol. 2018;17(11):1016-24.

73. Tarasoff-Conway JM, Carare RO, Osorio RS, Glodzik L, Butler T, Fieremans E, et al. Clearance systems in the brain-implications for Alzheimer disease. Nat Rev Neurol. 2015;11(8):457-70.

74. Fussi N, Hollerhage M, Chakroun T, Nykanen NP, Rosler TW, Koeglsperger T, et al. Exosomal secretion of alpha-synuclein as protective mechanism after upstream blockage of macroautophagy. Cell Death Dis. 2018;9(7):757.

75. Miranda AM, Lasiecka ZM, Xu Y, Neufeld J, Shahriar S, Simoes S, et al. Neuronal lysosomal dysfunction releases exosomes harboring APP C-terminal fragments and unique lipid signatures. Nat Commun. 2018;9(1):291.

76. Miranda AM DPG. Endolysosomal dysfunction and exosome secretion: implications for neurodegenerative disorders. Cell Stress. 2018;2(5):4. 
77. Ngolab J, Trinh I, Rockenstein E, Mante M, Florio J, Trejo M, et al. Brain-derived exosomes from dementia with Lewy bodies propagate alpha-synuclein pathology. Acta Neuropathol Commun. 2017;5(1):46.

78. Trotta T, Panaro MA, Cianciulli A, Mori G, Di Benedetto A, Porro C. Microglia-derived extracellular vesicles in Alzheimer's Disease: A double-edged sword. Biochem Pharmacol. 2018;148:18492.

79. Sweeney MD, Zhao Z, Montagne A, Nelson AR, Zlokovic BV. Blood-brain barrier: From physiology to disease and back. Physiol Rev. 2019;99(1):21-78.

80. Itkin T, Gur-Cohen S, Spencer JA, Schajnovitz A, Ramasamy SK, Kusumbe AP, et al. Distinct bone marrow blood vessels differentially regulate haematopoiesis. Nature. 2016;532(7599):323-8.

81. Cataldi M, Vigliotti C, Mosca T, Cammarota M, Capone D. Emerging role of the spleen in the pharmacokinetics of monoclonal antibodies, nanoparticles and exosomes. Int J Mol Sci. 2017;18(6).

82. Gangadaran P, Hong CM, Oh JM, Rajendran RL, Kalimuthu S, Son SH, et al. In vivo non-invasive imaging of radio-labeled exosome-mimetics derived from red blood cells in mice. Front Pharmacol. 2018;9:817.

83. Lai CP, Mardini O, Ericsson M, Prabhakar S, Maguire C, Chen JW, et al. Dynamic biodistribution of extracellular vesicles in vivo using a multimodal imaging reporter. ACS Nano. 2014;8(1):483-94.

84. Ohno S, Takanashi M, Sudo K, Ueda S, Ishikawa A, Matsuyama N, et al. Systemically injected exosomes targeted to EGFR deliver antitumor microRNA to breast cancer cells. Mol Ther. 2013;21(1):18591.

85. Peinado H, Aleckovic M, Lavotshkin S, Matei I, Costa-Silva B, Moreno-Bueno G, et al. Melanoma exosomes educate bone marrow progenitor cells toward a pro-metastatic phenotype through MET. Nat Med. 2012;18(6):883-91.

86. Krenkel O, Tacke F. Liver macrophages in tissue homeostasis and disease. Nat Rev Immunol. 2017;17(5):306-21.

87. Poisson J, Lemoinne S, Boulanger C, Durand F, Moreau R, Valla D, et al. Liver sinusoidal endothelial cells: Physiology and role in liver diseases. J Hepatol. 2017;66(1):212-27.

88. Shetty S, Lalor PF, Adams DH. Liver sinusoidal endothelial cells - gatekeepers of hepatic immunity. Nat Rev Gastroenterol Hepatol. 2018;15(9):555-67.

89. Imai T, Takahashi Y, Nishikawa M, Kato K, Morishita M, Yamashita T, et al. Macrophagedependent clearance of systemically administered B16BL6-derived exosomes from the blood circulation in mice. J Extracell Vesicles. 2015;4:26238.

90. Pillai S, Cariappa A. The follicular versus marginal zone B lymphocyte cell fate decision. Nat Rev Immunol. 2009;9(11):767-77.

91. Arenaccio C, Chiozzini C, Ferrantelli F, Leone P, Olivetta E, Federico M. Exosomes in Therapy: Engineering, pharmacokinetics and future applications. Curr Drug Targets. 2019;20(1):87-95.

92. Hadjidemetriou M, Al-Ahmady Z, Mazza M, Collins RF, Dawson K, Kostarelos K. In vivo biomolecule corona around blood-circulating, clinically used and antibody-targeted lipid bilayer nanoscale vesicles. ACS Nano. 2015;9(8):8142-56.

93. Sutaria DS, Jiang J, Elgamal OA, Pomeroy SM, Badawi M, Zhu X, et al. Low active loading of cargo into engineered extracellular vesicles results in inefficient miRNA mimic delivery. J Extracell Vesicles. 2017;6(1):1333882.

94. Wang X, Zhang H, Yang H, Bai M, Ning T, Li S, et al. Cell-derived exosomes as promising carriers for drug delivery and targeted therapy. Curr Cancer Drug Targets. 2018;18(4):347-54.

95. Hong SY, Tobias G, Al-Jamal KT, Ballesteros B, Ali-Boucetta H, Lozano-Perez S, et al. Filled and glycosylated carbon nanotubes for in vivo radioemitter localization and imaging. Nat Mater. 2010;9(6):48590.

96. Schubert J, Chanana M. Coating matters: Review on colloidal stability of nanoparticles with biocompatible coatings in biological media, living cells and organisms. Curr Med Chem. 2018;25(35):455386. 
97. Capriotti AL, Cavaliere C, Piovesana S. Liposome protein corona characterization as a new approach in nanomedicine. Anal Bioanal Chem. 2019.

98. Rezaei G, Daghighi SM, Haririan I, Yousefi I, Raoufi M, Rezaee F, et al. Protein corona variation in nanoparticles revisited: A dynamic grouping strategy. Colloids Surf B Biointerfaces. 2019;179:505-16.

99. Hood JL, Scott MJ, Wickline SA. Maximizing exosome colloidal stability following electroporation. Anal Biochem. 2014;448:41-9.

100. Linares R, Tan S, Gounou C, Arraud N, Brisson AR. High-speed centrifugation induces aggregation of extracellular vesicles. J Extracell Vesicles. 2015;4:29509.

101. Gonda A, Kabagwira J, Senthil GN, Wall NR. Internalization of exosomes through receptormediated endocytosis. Mol Cancer Res. 2019;17(2):337-47.

102. Emam SE, Ando H, Lila ASA, Shimizu T, Okuhira K, Ishima Y, et al. Liposome co-incubation with cancer cells secreted exosomes (extracellular vesicles) with different proteins expressions and different uptake pathways. Sci Rep. 2018;8(1):14493.

103. Chen W, Guo Y, Yang W, Chen L, Ren D, Wu C, et al. Phosphorylation of connexin 43 induced by traumatic brain injury promotes exosome release. J Neurophysiol. 2018;119(1):305-11.

104. Hamidi H, Ivaska J. Every step of the way: integrins in cancer progression and metastasis. Nat Rev Cancer. 2018;18(9):533-48.

105. Park EJ, Prajuabjinda O, Soe ZY, Darkwah S, Appiah MG, Kawamoto E, et al. Exosomal regulation of lymphocyte homing to the gut. Blood Adv. 2019;3(1):1-11.

106. Shattil SJ, Kim C, Ginsberg MH. The final steps of integrin activation: the end game. Nat Rev Mol Cell Biol. 2010;11(4):288-300.

107. Shimaoka M, Kawamoto E, Gaowa A, Okamoto T, Park EJ. Connexins and integrins in exosomes. Cancers (Basel). 2019;11(1).

108. Singh A, Fedele C, Lu H, Nevalainen MT, Keen JH, Languino LR. Exosome-mediated transfer of alphavbeta3 integrin from tumorigenic to nontumorigenic cells promotes a migratory phenotype. Mol Cancer Res. 2016;14(11):1136-46.

109. Soares AR, Martins-Marques T, Ribeiro-Rodrigues T, Ferreira JV, Catarino S, Pinho MJ, et al. Gap junctional protein $\mathrm{Cx} 43$ is involved in the communication between extracellular vesicles and mammalian cells. Sci Rep. 2015;5:13243.

110. Varela-Eirin M, Varela-Vazquez A, Rodriguez-Candela Mateos M, Vila-Sanjurjo A, Fonseca E, Mascarenas JL, et al. Recruitment of RNA molecules by connexin RNA-binding motifs: Implication in RNA and DNA transport through microvesicles and exosomes. Biochim Biophys Acta Mol Cell Res. 2017;1864(4):728-36.

111. Hoshino A, Costa-Silva B, Shen TL, Rodrigues G, Hashimoto A, Tesic Mark M, et al. Tumour exosome integrins determine organotropic metastasis. Nature. 2015;527(7578):329-35.

112. Triantafyllou E, Woollard KJ, McPhail MJW, Antoniades CG, Possamai LA. The role of monocytes and macrophages in acute and acute-on-chronic liver failure. Front Immunol. 2018;9:2948.

113. Sancho-Albero M, Navascues N, Mendoza G, Sebastian V, Arruebo M, Martin-Duque P, et al. Exosome origin determines cell targeting and the transfer of therapeutic nanoparticles towards target cells. J Nanobiotechnology. 2019;17(1):16.

114. Anand S, Samuel M, Kumar S, Mathivanan S. Ticket to a bubble ride: Cargo sorting into exosomes and extracellular vesicles. Biochim Biophys Acta Proteins Proteom. 2019.

115. Cypryk W, Nyman TA, Matikainen S. From inflammasome to exosome-does extracellular vesicle secretion constitute an inflammasome-dependent immune response? Front Immunol. 2018;9:2188.

116. Pleet ML, Branscome H, DeMarino C, Pinto DO, Zadeh MA, Rodriguez M, et al. Autophagy, EVs, and infections: A perfect question for a perfect time. Front Cell Infect Microbiol. 2018;8:362.

117. Xu J, Camfield R, Gorski SM. The interplay between exosomes and autophagy - partners in crime. J Cell Sci. 2018;131(15).

118. Chen L, Chen R, Kemper S, Brigstock DR. Pathways of production and delivery of hepatocyte exosomes. J Cell Commun Signal. 2018;12(1):343-57. 
119. Morales-Prieto DM, Stojiljkovic M, Diezel C, Streicher P-E, Röstel F, Lindner J, et al. Peripheral blood exosomes pass blood-brain-barrier and induce glial cell activation. 2018:471409.

120. Laulagnier K, Javalet C, Hemming FJ, Chivet M, Lachenal G, Blot B, et al. Amyloid precursor protein products concentrate in a subset of exosomes specifically endocytosed by neurons. Cell Mol Life Sci. 2018;75(4):757-73.

121. Oh HJ, Shin Y, Chung S, Hwang DW, Lee DS. Convective exosome-tracing microfluidics for analysis of cell-non-autonomous neurogenesis. Biomaterials. 2017;112:82-94.

122. Nedergaard M. Neuroscience. Garbage truck of the brain. Science. 2013;340(6140):1529-30.

123. Wang Y, Balaji V, Kaniyappan S, Kruger L, Irsen S, Tepper K, et al. The release and trans-synaptic transmission of Tau via exosomes. Mol Neurodegener. 2017;12(1):5.

124. Fang Y, Dong Y, Zheng T, Du D, Wen J, Gao D, et al. Altered tracer distribution and clearance in the extracellular space of the substantia nigra in a rodent model of Parkinson's disease. Front Neurosci. 2017;11:409.

125. Dikic I, Elazar Z. Mechanism and medical implications of mammalian autophagy. Nat Rev Mol Cell Biol. 2018;19(6):349-64.

126. Benedetto R, Massicano AVF, Crenshaw BK, Oliveira R, Reis RM, Araujo EB, et al. (89)Zr-DFOCetuximab as a molecular imaging agent to identify cetuximab resistance in head and neck squamous cell carcinoma. Cancer Biother Radiopharm. 2019.

127. Rinne SS, Leitao CD, Mitran B, Bass TZ, Andersson KG, Tolmachev V, et al. Optimization of HER3 expression imaging using affibody molecules: Influence of chelator for labeling with indium-111. Sci Rep. 2019;9(1):655.

128. Rylova SN, Del Pozzo L, Klingeberg C, Tonnesmann R, Illert AL, Meyer PT, et al. Immuno-PET imaging of CD30-positive lymphoma using 89Zr-desferrioxamine-labeled CD30-specific AC-10 antibody. J Nucl Med. 2016;57(1):96-102.

129. Suh M, Lee DS. Brain theranostics and radiotheranostics: Exosomes and graphenes in vivo as novel brain theranostics. Nucl Med Mol Imaging. 2018;52(6):407-19.

130. Luk KC, Kehm VM, Zhang B, O'Brien P, Trojanowski JQ, Lee VM. Intracerebral inoculation of pathological alpha-synuclein initiates a rapidly progressive neurodegenerative alpha-synucleinopathy in mice. J Exp Med. 2012;209(5):975-86.

131. Yang L, Sundaresan G, Sun M, Jose P, Hoffman D, McDonagh PR, et al. Intrinsically radiolabeled multifunctional cerium oxide nanoparticles for in vivo studies. J Mater Chem B. 2013;1(10):1421-31. 